\title{
Stabilised finite element methods for a bending moment formulation of the Reissner-Mindlin plate model *
}

\author{
Gabriel R. Barrenechea ${ }^{\dagger}$ Tomás P. Barrios $\ddagger$ and Andreas Wachtel
}

\begin{abstract}
This work presents new stabilised finite element methods for a bending moments formulation of the Reissner-Mindlin plate model. The introduction of the bending moment as an extra unknown leads to a new weak formulation, where the symmetry of this variable is imposed strongly in the space. This weak problem is proved to be well-posed, and stabilised Galerkin schemes for its discretisation are presented and analysed. The finite element methods are such that the bending moment tensor is sought in a finite element space constituted of piecewise linear continuos and symmetric tensors. Optimal error estimates are proved, and these findings are illustrated by representative numerical experiments.
\end{abstract}

\section{Mathematics Subject Classifications (1991):}

Key words: Reissner-Mindlin plate; stabilised finite element method; symmetric formulation; symmetric tensor.

\section{Introduction}

The Reissner-Mindlin equations are widely used by engineers to describe the behaviour of an elastic plate loaded by a transverse force. In this model the shear deformations are taken into account. Then, the theory is applicable to both thin and moderately thick plates, for which the normal to the mid-surface remains straight but not necessarily perpendicular to the mid-surface.

One particularity of these equations is that the thickness of the plate appears explicitly in the partial differential equations. Then, if these equations are discretised using standard piecewise polynomial spaces, numerical locking appears. With the aim of avoiding this locking (i.e., in order to

\footnotetext{
* This research was partially supported by the Leverhulme Trust through the Research Grant Project No. RPG-2012483. The work of TB was also partially funded by Dirección de Investigación de Universidad Católica de la Santísima Concepción, Chile.

${ }^{\dagger}$ Department of Mathematics and Statistics, University of Strathclyde, 26 Richmond Street, Glasgow G1 1XH, Scotland, email: gabriel.barrenechea@strath.ac.uk

${ }^{\ddagger}$ Departamento de Matemática y Física Aplicadas, Universidad Católica de la Santísima Concepción, Casilla 297, Concepción, Chile, email: tomas@ucsc.cl

${ }^{\S}$ Department of Mathematics and Statistics, University of Strathclyde, 26 Richmond Street, Glasgow G1 1XH, Scotland, email: andreas.wachtel@strath.ac.uk
} 
design finite element methods whose error estimates are independent of the thickness of the plate) a considerable amount of work has been produced over the last couple of decades. Most of these works have been carried out using a mixed finite element approach, see, e.g., [2], [12], [19], [1], and [14] for an overview, and the references therein. All these references deal with a second order equation for the rotations, while the remaining variables were determined by first-order partial differential equations. As an alternative, recently there has been an interest in developing finite element methods based on a rewriting of the equations as a first order system introducing the bending moment tensor as an extra unknown. For example, in [3] the problem is posed in terms of six variables lying in $L^{2}$ and $H($ div) spaces, and then the discrete system is hybridised. In [4], the bending moment has been introduced as an auxiliary unknown, the symmetry of this tensor has been imposed using a Lagrange multiplier and the discretisation has been carried out with the aid of PEERS elements.

All of the approaches quoted above need the introduction of inf-sup stable pairs of elements for which the symmetry of the stress tensor is not easily imposed. This is, in fact, the motivation for introducing a Lagrange multiplier in [4], and for the introduction of the anti-symmetric gradient as an extra unknown in [3]. This symmetry is easier to impose if the variables are sought in spaces of complete polynomials (rather than Raviart-Thomas like spaces). These spaces are not, in general, inf-sup stable, especially for the lowest order, and then some stabilisation is needed. Unlike the infsup stable framework, the number of stabilised finite element methods for the bending plate problem using standard nodal elements is, up to our knowledge, low. For example, in [17] the original weak formulation is enhanced with least-squares type terms and the analysis performed for high order polynomials. In [22] a simpler variant of this method, allowing the lowest polynomial order, is proposed and analysed. In [8] an interpolation of the test functions into the lowest order Raviart-Thomas space is used within the least-squares term, thus making this method only usable for first order elements. A recent stabilised alternative, using the same unknowns, has been developed in [18]. Finally, we mention the work [21] where a refined analysis of a family of stabilised methods and a multi grid algorithm are proposed. It is worth mentioning that none of these references deal with the system of first order equations, and then the bending moment tensor needs to be computed as the symmetric part of the gradient of the displacement vector multiplied by the appropriate physical constants.

The aim of this work is then to propose a stabilised formulation for the Reissner-Mindlin problem written as a system of four first order differential equations, having the bending moment tensor as an extra unknown. The main interest is to discretise the bending moment tensor using a finite element space of symmetric complete polynomials of first order. This leads to an inf-sup deficiency that is compensated for by designing appropriate stabilising terms. Our interest in this work is to alter the Galerkin formulation as little as possible, and then this inf-sup deficiency has to be characterised precisely before proposing the method.

The rest of this manuscript is organised as follows. The remaining of this introduction states the main notations and the problem of interest. In Section 2 the continuous problem is analysed, and its well-posedness is proved. Section 3 starts by analysing the inf-sup deficiency of the lowest order pair of finite element spaces. This information is used to propose a stabilised finite element method, whose stability is proved and optimal error estimates are obtained. Section 4 extends these findings to the case in which the finite element spaces used are all of equal order. Finally, in Section 5 some numerical results illustrating our findings are presented. 


\subsection{Notations and the problem of interest}

Given $\boldsymbol{\tau}:=\left(\tau_{i j}\right), \boldsymbol{\zeta}:=\left(\zeta_{i j}\right) \in \mathbb{R}^{2 \times 2}$, we write, as usual, $\boldsymbol{\tau}^{\mathrm{t}}:=\left(\tau_{j i}\right), \operatorname{tr}(\boldsymbol{\tau}):=\tau_{11}+\tau_{22}$ and $\boldsymbol{\tau}: \boldsymbol{\zeta}:=\sum_{i, j=1}^{2} \tau_{i j} \zeta_{i j}$. We will use standard notation for Sobolev spaces. In particular, $(\cdot, \cdot)_{D}$ stands for the $L^{2}(D)$ inner product, $\|\cdot\|_{r, D}$ and $|\cdot|_{r, D}$ denote the norm and seminorm in $H^{r}(D)$, respectively, where $H^{0}(D)=L^{2}(D)$, and no distinction is made for vector or tensor-valued functions. For $\boldsymbol{v}=\left(v_{1}, v_{2}\right)$ and $\boldsymbol{\tau}=\left(\tau_{i j}\right)$ we define the following differential operators

$$
\operatorname{div}(\boldsymbol{v}):=\partial_{x} v_{1}+\partial_{y} v_{2} \quad, \quad \operatorname{div}(\boldsymbol{\tau}):=\left[\begin{array}{c}
\partial_{x} \tau_{11}+\partial_{y} \tau_{12} \\
\partial_{x} \tau_{21}+\partial_{y} \tau_{22}
\end{array}\right]
$$

and, for $D \subset \mathbb{R}^{2}$ we define the function spaces

$$
\begin{aligned}
H(\operatorname{div}, D) & :=\left\{\boldsymbol{v} \in L^{2}(D)^{2}: \operatorname{div}(\boldsymbol{v}) \in L^{2}(D)\right\}, \\
H(\operatorname{div}, D) & :=\left\{\boldsymbol{\tau} \in L^{2}(D)^{2 \times 2}: \operatorname{div}(\boldsymbol{\tau}) \in L^{2}(D)^{2}\right\},
\end{aligned}
$$

equipped with their usual norms

$$
\|\boldsymbol{v}\|_{\operatorname{div}, D}^{2}:=\|\boldsymbol{v}\|_{0, D}^{2}+\|\operatorname{div}(\boldsymbol{v})\|_{0, D}^{2} \quad \text { and } \quad\|\boldsymbol{\tau}\|_{\operatorname{div}, D}^{2}:=\|\boldsymbol{\tau}\|_{0, D}^{2}+\|\operatorname{div}(\boldsymbol{\tau})\|_{0, D}^{2} .
$$

Finally, if the boundary of $D$ is denoted by $\partial D,\langle\cdot, \cdot\rangle_{\partial D}$ stands for the duality pairing between $H^{-\frac{1}{2}}(\partial D)$ and $H^{\frac{1}{2}}(\partial D)$.

The physical domain (the plate) is the cylindrical domain $\Omega \times\left(-\frac{t}{2}, \frac{t}{2}\right)$, where $\Omega \subseteq \mathbb{R}^{2}$ is an open polygonal domain, and $0<t \ll \operatorname{diam}(\Omega)$. Then, given a source term $g \in L^{2}(\Omega)$, the Reissner-Mindlin model seeks for rotations $\boldsymbol{\beta}=\left(\beta_{1}, \beta_{2}\right)$ of the fybres initially normal to the plate's midsurface, the scaled shear stress $\gamma=\left(\gamma_{1}, \gamma_{2}\right)$ and the transversal displacement $\omega$, such that

$$
\begin{aligned}
-\operatorname{div}(\mathcal{C}(\varepsilon(\boldsymbol{\beta})))-\boldsymbol{\gamma}=\mathbf{0} & \text { in } \quad \Omega, \\
-\operatorname{div}(\boldsymbol{\gamma})=g & \text { in } \quad \Omega, \\
\boldsymbol{\gamma}-\frac{\kappa}{t^{2}}(\nabla \omega-\boldsymbol{\beta})=\mathbf{0} & \text { in } \quad \Omega, \\
\omega=0, \boldsymbol{\beta}=\mathbf{0} & \text { on } \quad \partial \Omega,
\end{aligned}
$$

where $\kappa:=E k / 2(1+\nu)$ is the shear modulus, $E$ the Young modulus, $\nu$ the Poisson ratio, and $k$ a correction factor usually taken as $5 / 6$ for clamped plates. Just to simplify the presentation we will suppose that $\frac{t^{2}}{\kappa} \leq 1$. As standard for isotropic materials, $\boldsymbol{\varepsilon}(\boldsymbol{\beta}):=\frac{1}{2}\left(\nabla \boldsymbol{\beta}+(\nabla \boldsymbol{\beta})^{\mathrm{t}}\right)$ denotes the strain tensor and $\mathcal{C}$ is the tensor of bending moduli, characterised by

$$
\mathcal{C} \boldsymbol{\tau}:=\frac{E}{12\left(1-\nu^{2}\right)}((1-\nu) \boldsymbol{\tau}+\nu \operatorname{tr}(\boldsymbol{\tau}) \boldsymbol{I}) \quad \forall \boldsymbol{\tau} \in L^{2}(\Omega)^{2 \times 2}
$$

where $\boldsymbol{I}$ is the identity matrix in $\mathbb{R}^{2 \times 2}$. Its inverse is given by

$$
\mathcal{C}^{-1} \boldsymbol{\tau}:=\frac{12\left(1-\nu^{2}\right)}{E}\left(\frac{1}{(1-\nu)} \boldsymbol{\tau}-\frac{\nu}{\left(1-\nu^{2}\right)} \operatorname{tr}(\boldsymbol{\tau}) \boldsymbol{I}\right) \quad \forall \boldsymbol{\tau} \in L^{2}(\Omega)^{2 \times 2} .
$$

We use $C$ or $c$, with or without subscripts, to denote generic constants, independent of the discretisation parameters and the thickness of the plate, which may take different values at different occurrences. Finally, every physical constant, with the exception of $t$, will be treated as a fixed constant. 


\section{The dual mixed formulation}

Following the idea recently proposed in [4], we introduce the bending moment $\boldsymbol{\sigma}:=\mathcal{C}(\boldsymbol{\varepsilon}(\boldsymbol{\beta}))$ in $\Omega$ as an additional unknown. Then, the first equation in (1) becomes

$$
\begin{aligned}
\mathcal{C}^{-1} \boldsymbol{\sigma}-\boldsymbol{\varepsilon}(\boldsymbol{\beta})=\mathbf{0} & \text { in } \quad \Omega, \\
-\boldsymbol{d i v}(\boldsymbol{\sigma})-\boldsymbol{\gamma}=\mathbf{0} & \text { in } \Omega .
\end{aligned}
$$

To present the new mixed formulation, the following function space will be useful

$$
\boldsymbol{H}:=\left\{\boldsymbol{\tau} \in H(\operatorname{div}, \Omega): \boldsymbol{\tau}^{t}=\boldsymbol{\tau} \text { in } \Omega\right\} .
$$

Since $\boldsymbol{\sigma}=\boldsymbol{\sigma}^{\mathrm{t}}$ in $\Omega$, then $\boldsymbol{\sigma} \in \boldsymbol{H}$. Next, multiplying (4) by $\boldsymbol{\tau} \in \boldsymbol{H}$ and (5) by $\boldsymbol{v} \in L^{2}(\Omega)^{2}$ and integrating by parts one arrives at

$$
\begin{array}{rlrl}
\int_{\Omega} \mathcal{C}^{-1} \boldsymbol{\sigma}: \boldsymbol{\tau}+\int_{\Omega} \boldsymbol{\beta} \cdot \operatorname{div}(\boldsymbol{\tau}) & =0 & & \forall \boldsymbol{\tau} \in \boldsymbol{H} \\
\int_{\Omega} \operatorname{div}(\boldsymbol{\sigma}) \cdot \boldsymbol{v}+\int_{\Omega} \boldsymbol{\gamma} \cdot \boldsymbol{v} & =0 & \forall \boldsymbol{v} \in L^{2}(\Omega)^{2} .
\end{array}
$$

In addition, multiplying the second equation of (1) by test functions $q \in H_{0}^{1}(\Omega)$ and integrating by parts gives

$$
-\int_{\Omega} \gamma \cdot \nabla q=-\int_{\Omega} g q \quad \forall q \in H_{0}^{1}(\Omega) .
$$

Finally, testing the third equation of (1) by test functions $\boldsymbol{z} \in L^{2}(\Omega)^{2}$ leads to

$$
-\int_{\Omega}(\nabla \omega-\boldsymbol{\beta}) \cdot \boldsymbol{z}+\frac{t^{2}}{\kappa} \int_{\Omega} \boldsymbol{\gamma} \cdot \boldsymbol{z}=0 \quad \forall \boldsymbol{z} \in L^{2}(\Omega)^{2} .
$$

Then, equations (7)-(10) can be gathered in the following weak formulation for (1): Find $(\boldsymbol{\sigma}, \boldsymbol{\beta}, \omega, \boldsymbol{\gamma}) \in$ $\mathcal{X}:=\boldsymbol{H} \times L^{2}(\Omega)^{2} \times H_{0}^{1}(\Omega) \times L^{2}(\Omega)^{2}$ such that

$$
A((\boldsymbol{\sigma}, \boldsymbol{\beta}, \omega, \boldsymbol{\gamma}),(\boldsymbol{\tau}, \boldsymbol{v}, q, \boldsymbol{z}))=-\int_{\Omega} g q \quad \forall(\boldsymbol{\tau}, \boldsymbol{v}, q, \boldsymbol{z}) \in \mathcal{X},
$$

where the bilinear form $A: \mathcal{X} \times \mathcal{X} \rightarrow \mathbb{R}$ is given by

$$
\begin{array}{r}
A((\boldsymbol{\sigma}, \boldsymbol{\beta}, \omega, \boldsymbol{\gamma}),(\boldsymbol{\tau}, \boldsymbol{v}, q, \boldsymbol{z}))=\int_{\Omega} \mathcal{C}^{-1} \boldsymbol{\sigma}: \boldsymbol{\tau}+\int_{\Omega} \boldsymbol{\beta} \cdot \operatorname{div}(\boldsymbol{\tau})+\int_{\Omega} \operatorname{div}(\boldsymbol{\sigma}) \cdot \boldsymbol{v} \\
-\int_{\Omega} \boldsymbol{\gamma} \cdot(\nabla q-\boldsymbol{v})-\int_{\Omega} \boldsymbol{z} \cdot(\nabla \omega-\boldsymbol{\beta})+\frac{t^{2}}{\kappa} \int_{\Omega} \boldsymbol{\gamma} \cdot \boldsymbol{z} .
\end{array}
$$

Remark 2.1 In the recent work [11] a different weak formulation was introduced for the same variables introduced above. The bilinear forms involved in that weak formulation are different, which induces a radically different choice of norms from the one presented in this work. In particular, the norms from [11] include the plate thickness $t$ and some dual norms, while our aim is to analyse the stability and convergence of approximations to (11) using the natural norms for the function spaces involved. 


\subsection{Well posedeness}

With the aim to establish the existence and uniqueness of the solution of the Problem (11), we recall the weak formulation for (1) introduced in [4]: Find $((\boldsymbol{\sigma}, \boldsymbol{\gamma}),(\boldsymbol{\beta}, r, \omega)) \in \tilde{\boldsymbol{H}} \times \boldsymbol{Q}$ such that

$$
\begin{aligned}
\int_{\Omega} \mathcal{C}^{-1} \boldsymbol{\sigma}: \boldsymbol{\tau}+\frac{t^{2}}{\kappa} \int_{\Omega} \boldsymbol{\gamma} \cdot \boldsymbol{\xi}+\int_{\Omega} \boldsymbol{\beta} \cdot(\operatorname{div}(\boldsymbol{\tau})+\boldsymbol{\xi})+\int_{\Omega} r\left(\boldsymbol{\tau}_{12}-\boldsymbol{\tau}_{21}\right)+\int_{\Omega} \omega \operatorname{div}(\boldsymbol{\xi}) & =0 \\
\int_{\Omega} \boldsymbol{\eta} \cdot(\operatorname{div}(\boldsymbol{\sigma})+\boldsymbol{\gamma})+\int_{\Omega} s\left(\boldsymbol{\sigma}_{12}-\boldsymbol{\sigma}_{21}\right)+\int_{\Omega} \nu \operatorname{div}(\boldsymbol{\gamma}) & =-\int_{\Omega} g \nu,
\end{aligned}
$$

for all $((\boldsymbol{\tau}, \boldsymbol{\xi}),(\boldsymbol{\eta}, s, \nu)) \in \tilde{\boldsymbol{H}} \times \boldsymbol{Q}$, where $\tilde{\boldsymbol{H}}:=H(\operatorname{div} ; \Omega) \times H(\operatorname{div}, \Omega)$ and $\boldsymbol{Q}:=L^{2}(\Omega)^{2} \times L^{2}(\Omega) \times L^{2}(\Omega)$. In [4] this last problem is proved to be well-posed, and the following regularity result stated: If $\Omega$ is a convex polygon, there exists a constant $C>0$, independent of $t$, such that

$$
\|\omega\|_{2, \Omega}+\|\boldsymbol{\beta}\|_{2, \Omega}+\|\boldsymbol{\gamma}\|_{\operatorname{div}, \Omega}+t\|\boldsymbol{\gamma}\|_{1, \Omega}+\|\boldsymbol{\sigma}\|_{1, \Omega}+t\|\operatorname{div} \boldsymbol{\sigma}\|_{1, \Omega}+\|r\|_{1, \Omega} \leq C\|g\|_{0, \Omega}
$$

In [4], Theorem 2.2, the well-posedness of (13) was stated assuming that $\Omega$ is a convex polygonal domain. Now, a careful reading of the proof of the results leading to Theorem 2.2 in [4] shows that the convexity assumption is not needed and then one can state that (13) is well-posed for general open polygonal plates. This fact will be used below since the following result states that every solution of (13) also solves (11).

Lemma 2.2 Let $((\boldsymbol{\sigma}, \boldsymbol{\gamma}),(\boldsymbol{\beta}, r, \omega)) \in \tilde{\boldsymbol{H}} \times \boldsymbol{Q}$ be the unique solution of (13). Then, $(\boldsymbol{\sigma}, \boldsymbol{\beta}, \omega, \boldsymbol{\gamma}) \in \mathcal{X}$ and solves (11).

Proof. Testing the second equation of (13) with $(\mathbf{0}, s, 0) \in \boldsymbol{Q}$ gives $\boldsymbol{\sigma}=\boldsymbol{\sigma}^{\text {t }}$ in $\Omega$, i.e., $\boldsymbol{\sigma} \in \boldsymbol{H}$. Furthermore, using $(\mathbf{0}, \boldsymbol{\xi}) \in \tilde{\boldsymbol{H}}$ as test function in the first equation of (13) gives

$$
\int_{\Omega}\left(\frac{t^{2}}{\kappa} \boldsymbol{\gamma}+\boldsymbol{\beta}\right) \cdot \boldsymbol{\xi}+\int_{\Omega} \omega \operatorname{div}(\boldsymbol{\xi})=0 \quad \forall \boldsymbol{\xi} \in H(\operatorname{div}, \Omega) .
$$

This implies that in the distributional sense $\nabla \omega=\frac{t^{2}}{\kappa} \boldsymbol{\gamma}+\boldsymbol{\beta} \in L^{2}(\Omega)^{2}$, and then $\omega \in H^{1}(\Omega)$. Hence, after integrating by parts in (15), it follows that $\langle\boldsymbol{\xi} \cdot \boldsymbol{\nu}, \omega\rangle_{\partial \Omega}=0$ on $\partial \Omega$, for all $\boldsymbol{\xi} \in H(\operatorname{div}, \Omega)$. The surjectivity of the normal trace implies $\omega=0$ on $\partial \Omega$ and thus $\omega \in H_{0}^{1}(\Omega)$. Therefore, $(\boldsymbol{\sigma}, \boldsymbol{\beta}, \omega, \gamma) \in \mathcal{X}$.

To prove that $(\boldsymbol{\sigma}, \boldsymbol{\beta}, \omega, \boldsymbol{\gamma})$ solves (11), first one notes that the first equation in (13) with $(\boldsymbol{\tau}, \mathbf{0})$, $\boldsymbol{\tau} \in \boldsymbol{H}$, as test function is (7). Also, using $(\mathbf{0}, \boldsymbol{\xi})$ as test function, integration by parts, and the density of $H(\operatorname{div}, \Omega)$ in $L^{2}(\Omega)^{2}$ leads to equation (10). Next, using integration by parts one can see that (9) is nothing but the second equation in (13) tested against $(\mathbf{0}, 0, \nu)$ where $\nu \in H_{0}^{1}(\Omega)$. Finally, (8) is recovered by restricting the second equation of (13) to test functions of the form $(\boldsymbol{\eta}, 0,0)$.

The uniqueness of solution of (11) will be established directly in the following lemma.

Lemma 2.3 Let $(\tilde{\boldsymbol{\sigma}}, \tilde{\boldsymbol{\beta}}, \tilde{\omega}, \tilde{\gamma}) \in \mathcal{X}$ be such that

$$
A((\tilde{\boldsymbol{\sigma}}, \tilde{\boldsymbol{\beta}}, \tilde{\omega}, \tilde{\gamma}),(\boldsymbol{\tau}, \boldsymbol{v}, q, \boldsymbol{z}))=0 \quad \forall(\boldsymbol{\tau}, \boldsymbol{v}, q, \boldsymbol{z}) \in \mathcal{X} .
$$

Then, $(\tilde{\boldsymbol{\sigma}}, \tilde{\boldsymbol{\beta}}, \tilde{\omega}, \tilde{\gamma})=(\mathbf{0}, \mathbf{0}, 0, \mathbf{0})$. 
Proof. From (3) it follows that

$$
\int_{\Omega} \mathcal{C}^{-1} \boldsymbol{\tau}: \boldsymbol{\tau} \geq \frac{12(1-\nu)}{E}\|\boldsymbol{\tau}\|_{0, \Omega}^{2} \quad \forall \boldsymbol{\tau} \in \boldsymbol{H}
$$

which gives

$$
A((\tilde{\boldsymbol{\sigma}}, \tilde{\boldsymbol{\beta}}, \tilde{\omega}, \tilde{\gamma}),(\tilde{\boldsymbol{\sigma}},-\tilde{\boldsymbol{\beta}},-\tilde{\omega}, \tilde{\boldsymbol{\gamma}})) \geq \frac{12(1-\nu)}{E}\|\tilde{\boldsymbol{\sigma}}\|_{0, \Omega}^{2}+\frac{t^{2}}{\kappa}\|\tilde{\boldsymbol{\gamma}}\|_{0, \Omega}^{2},
$$

and then $\tilde{\boldsymbol{\sigma}}=\mathbf{0}$ and $\tilde{\boldsymbol{\gamma}}=\mathbf{0}$. Next, let $\tilde{\boldsymbol{\tau}}:=\boldsymbol{\varepsilon}(\boldsymbol{u})$, where $\boldsymbol{u} \in H^{1}(\Omega)^{2}$ is the unique solution of

$$
\operatorname{div}(\varepsilon(\boldsymbol{u}))=\tilde{\boldsymbol{\beta}} \quad \text { in } \Omega \quad, \quad \boldsymbol{u}=\mathbf{0} \quad \text { on } \partial \Omega .
$$

Then, $\tilde{\boldsymbol{\tau}} \in \boldsymbol{H}$ and

$$
A((\mathbf{0}, \tilde{\boldsymbol{\beta}}, \tilde{\omega}, \mathbf{0}),(\tilde{\boldsymbol{\tau}}, \mathbf{0}, 0, \mathbf{0}))=\|\tilde{\boldsymbol{\beta}}\|_{0, \Omega}^{2}=0
$$

which gives $\tilde{\boldsymbol{\beta}}=\mathbf{0}$. Finally,

$$
A((\mathbf{0}, 0, \tilde{\omega}, \mathbf{0}),(\mathbf{0}, \mathbf{0}, 0,-\nabla \tilde{\omega}))=\|\nabla \tilde{\omega}\|_{0, \Omega}^{2}=0,
$$

which shows that $\tilde{\omega}=0$.

The previous findings can be summarised in the following theorem.

Theorem 2.4 There exists a unique $(\boldsymbol{\sigma}, \boldsymbol{\beta}, \omega, \boldsymbol{\gamma}) \in \mathcal{X}$ solution of (11). In addition, if $\Omega$ is convex, it satisfies (14).

From a discrete point of view, the bilinear form $A$ associated to (11) is not elliptic in the whole space, and then care must be paid to the choice of the finite element spaces used to discretise this problem. As was mentioned in the introduction, the most commonly used spaces to present a stable discrete method are of Raviart-Thomas, or PEERS, type. This led naturally to the introduction of the extra variable $r$ to impose this symmetry weakly. Our aim in this work is to take an alternative route, and then approximate $\boldsymbol{\sigma}$ in the space of piecewise linear continuos and symmetric tensors. Then, if $\omega$ is also discretised using piecewise linear continuous elements and $\boldsymbol{\beta}$ and $\boldsymbol{\gamma}$ are discretised using piecewise constants, some stabilisation must be used in order to obtain a well-posed problem. A desirable property of such a method is to add to the formulation as few extra terms as possible, in the spirit of the minimal stabilisation approach proposed in [9]. Like that, the discrete solution may be expected to inherit at least some of the properties of the continuous one. Another desirable property for a finite element method for this problem is a stability result, and hopefully error estimates, independent of the thickness of the plate. In the next section we will introduce a stabilised method satisfying these restrictions.

\section{The stabilised Galerkin scheme}

Let $\left\{\mathcal{T}_{h}\right\}_{h>0}$ be a regular family of triangulations of $\bar{\Omega}$. Given a triangle $T \in \mathcal{T}_{h}$, we denote by $h_{T}$ its diameter and define the mesh size $h:=\max \left\{h_{T}: T \in \mathcal{T}_{h}\right\}$. An interior edge of $\mathcal{T}_{h}$ is the (nonempty) 
interior of $\partial T \cap \partial T^{\prime}$, where $T$ and $T^{\prime}$ are two adjacent elements of $\mathcal{T}_{h}$. Similarly, a boundary edge of $\mathcal{T}_{h}$ is the interior of $\partial T \cap \partial \Omega$, if it is nonempty. We denote by $\mathcal{E}_{I}$ the set of all interior edges of $\mathcal{T}_{h}$, and by $\mathcal{E}$ the set of all edges of the triangulation, including the boundary. The boundary edges are denoted by $\mathcal{E}_{b}=\mathcal{E} \backslash \mathcal{E}_{I}$. Further, for each $e \in \mathcal{E}, h_{e}$ represents its length. For every edge $e \in \mathcal{E}$, we choose one unit normal vector $\boldsymbol{n}_{e}$ to $e$, pointing towards the exterior of $\Omega$ if $e \subset \partial \Omega$. Also, for $e \in \mathcal{E}$ and $T \in \mathcal{T}_{h}$ we introduce the neighbourhoods

$$
\omega_{e}=\cup\left\{T \in \mathcal{T}_{h}: T \cap e \neq \emptyset\right\} \quad, \quad \omega_{T}=\cup\left\{T^{\prime} \in \mathcal{T}_{h}: T^{\prime} \cap T \neq \emptyset\right\}
$$

In addition, let $\boldsymbol{v}$ be a vector-valued function, smooth inside each element $T \in \mathcal{T}_{h}$. We denote by $\boldsymbol{v}_{T, e}$ the restriction of $\boldsymbol{v}_{T}$ to $e$ and define the jump of $\boldsymbol{v}$ across it by:

$$
\llbracket \boldsymbol{v} \rrbracket:= \begin{cases}\boldsymbol{v}_{T, e}-\boldsymbol{v}_{T^{\prime}, e} & \text { if } e \in \mathcal{E}_{I}, \\ \boldsymbol{v}_{T, e} & \text { if } e \in \mathcal{E}_{b} .\end{cases}
$$

Let $e \in \mathcal{E}$ and $T \in \omega_{e}$. We recall the following local trace inequality: there exists $C>0$, independent of $h$, such that

$$
\|q\|_{0, e} \leq C\left(h_{e}^{-\frac{1}{2}}\|q\|_{0, T}+h_{e}^{\frac{1}{2}}|q|_{1, T}\right) \quad \forall q \in H^{1}(T) .
$$

Let the following piecewise polynomial spaces

$$
\begin{aligned}
& X_{h, 1}:=\left\{v_{h} \in C^{0}(\bar{\Omega}):\left.v_{h}\right|_{T} \in \mathbb{P}_{1}(T), \forall T \in \mathcal{T}_{h}\right\}, \\
& X_{h, 0}:=\left\{v_{h} \in L^{2}(\Omega):\left.v_{h}\right|_{T} \in \mathbb{P}_{0}(T), \forall T \in \mathcal{T}_{h}\right\},
\end{aligned}
$$

where given an integer $\ell \geq 0$ and a subset $D$ of $\mathbb{R}^{2}, \mathbb{P}_{\ell}(D)$ denotes the space of polynomials in two variables defined in $D$ of total degree at most $\ell$. Then, we define the following finite element spaces

$$
\begin{aligned}
H_{h}^{\boldsymbol{\sigma}} & :=\left\{\boldsymbol{\tau}_{h} \in X_{h, 1}^{2 \times 2}: \boldsymbol{\tau}_{h}=\boldsymbol{\tau}_{h}^{\mathrm{t}} \quad \text { in } \Omega\right\}, \\
H_{h}^{\boldsymbol{\beta}}=H_{h}^{\boldsymbol{\gamma}} & :=X_{h, 0}^{2}, \\
H_{h}^{\omega} & :=X_{h, 1} \cap H_{0}^{1}(\Omega), \\
\mathcal{Y}_{h} & :=H_{h}^{\boldsymbol{\sigma}} \times H_{h}^{\boldsymbol{\beta}} \times H_{h}^{\omega},
\end{aligned}
$$

and

$$
\mathcal{X}_{h}:=\mathcal{Y}_{h} \times H_{h}^{\gamma} .
$$

In what follows, we recall appropriate interpolation operators. Let $I_{h} \in \mathcal{L}\left(H^{1}(\Omega), X_{h, 1}\right) \cap \mathcal{L}\left(H_{0}^{1}(\Omega), X_{h, 1} \cap\right.$ $\left.H_{0}^{1}(\Omega)\right)$ be the Scott-Zhang interpolation operator. This interpolator satisfies the following properties (cf. [13]): for $0 \leq m \leq 1$ and $1 \leq n \leq 2$,

$$
\begin{aligned}
& \left\|v-I_{h} v\right\|_{m, T} \leq C h_{T}^{n-m}|v|_{n, \omega_{T}} \quad \forall v \in H^{n}\left(\omega_{T}\right), \\
& \left\|v-I_{h} v\right\|_{m, e} \leq C h_{e}^{n-m-\frac{1}{2}}|v|_{n, \omega_{e}} \quad \forall v \in H^{n}\left(\omega_{e}\right),
\end{aligned}
$$


where $C>0$ is independent of $h$. The orthogonal projection $\Pi_{h, \ell}: L^{2}(\Omega) \rightarrow X_{h, \ell}, \ell=0,1$, defined by

$$
\int_{\Omega}\left(v-\Pi_{h, \ell}(v)\right) w_{h}=0 \quad \forall w_{h} \in X_{h, \ell},
$$

will be useful later on as well. This projection satisfies the approximation properties (cf. [13]): for $0 \leq j \leq k \leq \ell+1$, and all $v \in H^{k}(\Omega)$, the following holds

$$
\begin{gathered}
\left\|v-\Pi_{h, \ell}(v)\right\|_{j, \Omega} \leq C h^{k-j}\|v\|_{k, \Omega}, \\
\left\{\sum_{e \in \mathcal{E}} h_{e}\left\|\llbracket v-\Pi_{h, 0}(v) \rrbracket\right\|_{0, e}^{2}\right\}^{\frac{1}{2}} \leq C h\|v\|_{1, \Omega}, \quad\left\{\sum_{e \in \mathcal{E}} h_{e}\left\|v-\Pi_{h, 1}(v)\right\|_{0, e}^{2}\right\}^{\frac{1}{2}} \leq C h^{k}\|v\|_{k, \Omega} .
\end{gathered}
$$

Finally, we mention that in the case of vector (or tensor)-valued functions these operators are taken component-wise.

\subsection{The inf-sup deficiency and the stabilised method}

In the next result we paraphrase the results in [5] and state the inf-sup deficiency of our choice of finite element spaces. This result will lead to the definition of the stabilised finite element scheme.

Lemma 3.1 For every $\boldsymbol{v}_{h} \in X_{h, 0}^{2}$, there exists $\tilde{\boldsymbol{\tau}}_{h} \in H_{h}^{\boldsymbol{\sigma}}$ such that

$$
\left\|\tilde{\boldsymbol{\tau}}_{h}\right\|_{1, \Omega} \leq C_{\text {sup }}\left\|\boldsymbol{v}_{h}\right\|_{0, \Omega}
$$

and

$$
\int_{\Omega} \operatorname{div}\left(\tilde{\boldsymbol{\tau}}_{h}\right) \cdot \boldsymbol{v}_{h} \geq \frac{1}{2}\left\|\boldsymbol{v}_{h}\right\|_{0, \Omega}^{2}-c_{i n f} \sum_{e \in \mathcal{E}} h_{e}\left\|\llbracket \boldsymbol{v}_{h} \rrbracket\right\|_{0, e}^{2},
$$

where the positive constants $C_{\text {sup }}$ and $c_{\text {inf }}$ are independent of $\boldsymbol{v}_{h}, \tilde{\boldsymbol{\tau}}_{h}, h$, and, in particular, $t$.

Proof. Let $\boldsymbol{v}_{h} \in X_{h, 0}^{2}$. Let $\hat{\Omega} \subseteq \mathbb{R}^{2}$ be an open, bounded, polygonal, and convex domain such that $\Omega \subseteq \hat{\Omega}$. Let $\boldsymbol{u} \in H^{1}(\hat{\Omega})^{2}$ be the unique solution of the problem

$$
-\operatorname{div}(\varepsilon(\boldsymbol{u}))=\hat{\boldsymbol{v}}_{h} \quad \text { in } \quad \hat{\Omega} \quad, \quad \boldsymbol{u}=\mathbf{0} \quad \text { on } \partial \hat{\Omega},
$$

where $\hat{\boldsymbol{v}}_{h}$ stands for the extension of $\boldsymbol{v}_{h}$ by zero to $\hat{\Omega} \backslash \Omega$. Since $\hat{\Omega}$ is convex, it follows that $\boldsymbol{u} \in H^{2}(\hat{\Omega})^{2}$. Defining $\tilde{\boldsymbol{\tau}}:=-\left.\boldsymbol{\varepsilon}(\boldsymbol{u})\right|_{\Omega} \in H^{1}(\Omega)^{2 \times 2}$ it follows that $\tilde{\boldsymbol{\tau}}=\tilde{\boldsymbol{\tau}}^{\mathrm{t}}$ in $\Omega$ and it satisfies

$$
\|\tilde{\boldsymbol{\tau}}\|_{1, \Omega} \leq c\left\|\boldsymbol{v}_{h}\right\|_{0, \Omega}
$$

where $c>0$ depends only on $\hat{\Omega}$. Next, let $\tilde{\boldsymbol{\tau}}_{h}:=I_{h}(\tilde{\boldsymbol{\tau}}) \in H_{h}^{\sigma}$ be the Scott-Zhang interpolate of $\tilde{\boldsymbol{\tau}}$. Then, (27) gives

$$
\left\|\tilde{\boldsymbol{\tau}}_{h}\right\|_{1, \Omega} \leq C\|\tilde{\boldsymbol{\tau}}\|_{1, \Omega} \leq C_{\text {sup }}\left\|\boldsymbol{v}_{h}\right\|_{0, \Omega}
$$


Moreover, using the Cauchy-Schwarz's and Young's inequalities, integration by parts, (28), (31), and the mesh regularity, one obtains

$$
\begin{aligned}
\left\|\boldsymbol{v}_{h}\right\|_{0, \Omega}^{2} & =\int_{\Omega} \boldsymbol{v}_{h} \cdot \operatorname{div}(\tilde{\boldsymbol{\tau}})=\int_{\Omega} \boldsymbol{v}_{h} \cdot \operatorname{div}\left(\tilde{\boldsymbol{\tau}}-\tilde{\boldsymbol{\tau}}_{h}\right)+\int_{\Omega} \boldsymbol{v}_{h} \cdot \operatorname{div}\left(\tilde{\boldsymbol{\tau}}_{h}\right) \\
& =\sum_{e \in \mathcal{E}} \int_{e} \llbracket \boldsymbol{v}_{h} \rrbracket \cdot\left(\tilde{\boldsymbol{\tau}}-\tilde{\boldsymbol{\tau}}_{h}\right) \boldsymbol{n}_{e}+\int_{\Omega} \boldsymbol{v}_{h} \cdot \operatorname{div}\left(\tilde{\boldsymbol{\tau}}_{h}\right) \\
& \leq \sum_{e \in \mathcal{E}}\left\|\llbracket \boldsymbol{v}_{h} \rrbracket\right\|_{0, e}\left\|\tilde{\boldsymbol{\tau}}-\tilde{\boldsymbol{\tau}}_{h}\right\|_{0, e}+\int_{\Omega} \boldsymbol{v}_{h} \cdot \operatorname{div}\left(\tilde{\boldsymbol{\tau}}_{h}\right) \\
& \leq C\left\{\sum_{e \in \mathcal{E}} h_{e}\left\|\llbracket \boldsymbol{v}_{h} \rrbracket\right\|_{0, e}^{2}\right\}^{1 / 2}\left\{\sum_{e \in \mathcal{E}}\|\tilde{\boldsymbol{\tau}}\|_{1, \omega_{e}}^{2}\right\}^{1 / 2}+\int_{\Omega} \boldsymbol{v}_{h} \cdot \operatorname{div}\left(\tilde{\boldsymbol{\tau}}_{h}\right) \\
& \leq c_{i n f} \sum_{e \in \mathcal{E}} h_{e}\left\|\llbracket \boldsymbol{v}_{h} \rrbracket\right\|_{0, e}^{2}+\frac{1}{2}\left\|\boldsymbol{v}_{h}\right\|_{0, \Omega}^{2}+\int_{\Omega} \boldsymbol{v}_{h} \cdot \operatorname{div}\left(\tilde{\boldsymbol{\tau}}_{h}\right),
\end{aligned}
$$

which, together with (32), completes the proof.

The previous result suggests the following stabilised finite element scheme associated to the variational formulation (11): Find $\left(\boldsymbol{\sigma}_{h}, \boldsymbol{\beta}_{h}, \omega_{h}, \gamma_{h}\right) \in \mathcal{X}_{h}$ such that

$$
A_{h}\left(\left(\boldsymbol{\sigma}_{h}, \boldsymbol{\beta}_{h}, \omega_{h}, \boldsymbol{\gamma}_{h}\right),\left(\boldsymbol{\tau}_{h}, \boldsymbol{v}_{h}, q_{h}, \boldsymbol{z}_{h}\right)\right)=-\int_{\Omega} g q_{h} \quad \forall\left(\boldsymbol{\tau}_{h}, \boldsymbol{v}_{h}, q_{h}, \boldsymbol{z}_{h}\right) \in \mathcal{X}_{h},
$$

where the bilinear form $A_{h}: \mathcal{X}_{h} \times \mathcal{X}_{h} \rightarrow \mathbb{R}$ is defined by

$$
A_{h}\left(\left(\boldsymbol{\sigma}_{h}, \boldsymbol{\beta}_{h}, \omega_{h}, \gamma_{h}\right),\left(\boldsymbol{\tau}_{h}, \boldsymbol{v}_{h}, q_{h}, \boldsymbol{z}_{h}\right)\right)=A\left(\left(\boldsymbol{\sigma}_{h}, \boldsymbol{\beta}_{h}, \omega_{h}, \gamma_{h}\right),\left(\boldsymbol{\tau}_{h}, \boldsymbol{v}_{h}, q_{h}, \boldsymbol{z}_{h}\right)\right)-\sum_{e \in \mathcal{E}} h_{e} \int_{e} \llbracket \boldsymbol{\beta}_{h} \rrbracket \cdot \llbracket \boldsymbol{v}_{h} \rrbracket .
$$

\subsection{Stability analysis}

In order to perform the analysis, the bilinear form $A_{h}$ is rewritten as follows:

$$
\begin{gathered}
A_{h}\left(\left(\boldsymbol{\sigma}_{h}, \boldsymbol{\beta}_{h}, \omega_{h}, \gamma_{h}\right),\left(\boldsymbol{\tau}_{h}, \boldsymbol{v}_{h}, q_{h}, \boldsymbol{z}_{h}\right)\right)=a_{h}\left(\left(\boldsymbol{\sigma}_{h}, \boldsymbol{\beta}_{h}, \omega_{h}\right),\left(\boldsymbol{\tau}_{h}, \boldsymbol{v}_{h}, q_{h}\right)\right) \\
+b\left(\left(\boldsymbol{\tau}_{h}, \boldsymbol{v}_{h}, q_{h}\right), \boldsymbol{\gamma}_{h}\right)+b\left(\left(\boldsymbol{\sigma}_{h}, \boldsymbol{\beta}_{h}, \omega_{h}\right), \boldsymbol{z}_{h}\right)+\frac{t^{2}}{\kappa} \int_{\Omega} \boldsymbol{\gamma}_{h} \cdot \boldsymbol{z}_{h},
\end{gathered}
$$

where $a_{h}: \mathcal{Y}_{h} \times \mathcal{Y}_{h} \rightarrow \mathbb{R}$ and $b: \mathcal{Y}_{h} \times H_{h}^{\gamma} \rightarrow \mathbb{R}$ are given by

$$
\begin{aligned}
a_{h}\left(\left(\boldsymbol{\sigma}_{h}, \boldsymbol{\beta}_{h}, \omega_{h}\right),\left(\boldsymbol{\tau}_{h}, \boldsymbol{v}_{h}, q_{h}\right)\right):= & \int_{\Omega} \mathcal{C}^{-1} \boldsymbol{\sigma}_{h}: \boldsymbol{\tau}_{h}+\int_{\Omega} \boldsymbol{\beta}_{h} \cdot \operatorname{div}\left(\boldsymbol{\tau}_{h}\right) \\
& +\int_{\Omega} \operatorname{div}\left(\boldsymbol{\sigma}_{h}\right) \cdot \boldsymbol{v}_{h}-\sum_{e \in \mathcal{E}} h_{e} \int_{e} \llbracket \boldsymbol{\beta}_{h} \rrbracket \cdot \llbracket \boldsymbol{v}_{h} \rrbracket \\
b\left(\left(\boldsymbol{\tau}_{h}, \boldsymbol{v}_{h}, q_{h}\right), \boldsymbol{z}_{h}\right):= & -\int_{\Omega} \boldsymbol{z}_{h} \cdot\left(\nabla q_{h}-\boldsymbol{v}_{h}\right) .
\end{aligned}
$$


The first step towards the stability result is an inf-sup condition for $b$. The appropriate norms on $H_{h}^{\boldsymbol{\beta}}$ and $\mathcal{Y}_{h}$ for this inf-sup condition are given now:

$$
\begin{gathered}
\left\|\boldsymbol{v}_{h}\right\|_{h, 0}^{2}:=\left\|\boldsymbol{v}_{h}\right\|_{0, \Omega}^{2}+\sum_{e \in \mathcal{E}} h_{e}\|\| \boldsymbol{v}_{h} \rrbracket \|_{0, e}^{2}, \\
\left\|\left(\boldsymbol{\tau}_{h}, \boldsymbol{v}_{h}, q_{h}\right)\right\|_{1}^{2}:=\left\|\boldsymbol{\tau}_{h}\right\|_{0, \Omega}^{2}+\left\|\boldsymbol{v}_{h}\right\|_{h, 0}^{2}+\left\|\nabla q_{h}\right\|_{0, \Omega}^{2},
\end{gathered}
$$

for all $\left(\boldsymbol{\tau}_{h}, \boldsymbol{v}_{h}, q_{h}\right) \in \mathcal{Y}_{h}$. The following result states the inf-sup condition for $b$.

Lemma 3.2 There exists $\tilde{\alpha}_{0}>0$, independent of $h$, such that

$$
\sup _{\left(\boldsymbol{\tau}_{h}, \boldsymbol{v}_{h}, q_{h}\right) \in \mathcal{Y}_{h} \backslash\{0\}} \frac{b\left(\left(\boldsymbol{\tau}_{h}, \boldsymbol{v}_{h}, q_{h}\right), \boldsymbol{z}_{h}\right)}{\left\|\left(\boldsymbol{\tau}_{h}, \boldsymbol{v}_{h}, q_{h}\right)\right\|_{1}} \geq \tilde{\alpha}_{0}\left\|\boldsymbol{z}_{h}\right\|_{0, \Omega},
$$

for all $\boldsymbol{z}_{h} \in H_{h}^{\gamma}$. Moreover, the discrete kernel of the bilinear form $b$ may be characterised as follows

$$
\begin{aligned}
\boldsymbol{V}_{h} & :=\left\{\left(\boldsymbol{\tau}_{h}, \boldsymbol{v}_{h}, q_{h}\right) \in \mathcal{Y}_{h}: b\left(\left(\boldsymbol{\tau}_{h}, \boldsymbol{v}_{h}, q_{h}\right), \boldsymbol{z}_{h}\right)=0 \forall \boldsymbol{z}_{h} \in H_{h}^{\gamma}\right\} \\
& =\left\{\left(\boldsymbol{\tau}_{h}, \boldsymbol{v}_{h}, q_{h}\right) \in \mathcal{Y}_{h}: \boldsymbol{v}_{h}=\nabla q_{h} \text { in } \Omega\right\} .
\end{aligned}
$$

Proof. First, using the local trace result (21) and the mesh regularity it follows that

$$
\left\|\boldsymbol{v}_{h}\right\|_{h, 0}^{2}=\left\|\boldsymbol{v}_{h}\right\|_{0, \Omega}^{2}+\sum_{e \in \mathcal{E}} h_{e}\left\|\llbracket \boldsymbol{v}_{h} \rrbracket\right\|_{0, e}^{2} \leq C\left\|\boldsymbol{v}_{h}\right\|_{0, \Omega}^{2}
$$

for all $\boldsymbol{v}_{h} \in H_{h}^{\boldsymbol{\beta}}$, where $C>0$ does not depend on $h$. Then, for all $\boldsymbol{z}_{h} \in H_{h}^{\gamma}$

$$
\sup _{\left(\boldsymbol{\tau}_{h}, \boldsymbol{v}_{h}, q_{h}\right) \in \mathcal{Y}_{h} \backslash\{0\}} \frac{b\left(\left(\boldsymbol{\tau}_{h}, \boldsymbol{v}_{h}, q_{h}\right), \boldsymbol{z}_{h}\right)}{\left\|\left(\boldsymbol{\tau}_{h}, \boldsymbol{v}_{h}, q_{h}\right)\right\|_{1}} \geq \sup _{\boldsymbol{v}_{h} \in H_{h}^{\boldsymbol{\beta}} \backslash\{0\}} \frac{\int_{\Omega} \boldsymbol{v}_{h} \cdot \boldsymbol{z}_{h}}{C\left\|\boldsymbol{v}_{h}\right\|_{0, \Omega}}=\tilde{\alpha}_{0}\left\|\boldsymbol{z}_{h}\right\|_{0, \Omega},
$$

which proves (40) with $\tilde{\alpha}_{0}:=C^{-1}$. The proof of the characterisation for $\boldsymbol{V}_{h}$ follows by noting that, for all $\left(\boldsymbol{\tau}_{h}, \boldsymbol{v}_{h}, q_{h}\right) \in \mathcal{Y}_{h}, \nabla q_{h}-\boldsymbol{v}_{h} \in H_{h}^{\gamma}$.

The next result states a uniform inf-sup condition for $a_{h}$.

Lemma 3.3 There exists $\tilde{\alpha}_{1}>0$, independent of $h$, such that for all $\left(\boldsymbol{\xi}_{h}, \boldsymbol{\varphi}_{h}, \mu_{h}\right) \in \boldsymbol{V}_{h}$ the following holds

$$
\sup _{\left(\boldsymbol{\tau}_{h}, \boldsymbol{v}_{h}, q_{h}\right) \in \boldsymbol{V}_{h} \backslash\{0\}} \frac{a_{h}\left(\left(\boldsymbol{\xi}_{h}, \boldsymbol{\varphi}_{h}, \mu_{h}\right),\left(\boldsymbol{\tau}_{h}, \boldsymbol{v}_{h}, q_{h}\right)\right)}{\left\|\left(\boldsymbol{\tau}_{h}, \boldsymbol{v}_{h}, q_{h}\right)\right\|_{1}} \geq \tilde{\alpha}_{1}\left\|\left(\boldsymbol{\xi}_{h}, \boldsymbol{\varphi}_{h}, \mu_{h}\right)\right\|_{1} .
$$

Proof. Let $\left(\boldsymbol{\xi}_{h}, \boldsymbol{\varphi}_{h}, \mu_{h}\right) \in \boldsymbol{V}_{h}$. From (16) and the definition of $a_{h}$ it follows that

$$
a_{h}\left(\left(\boldsymbol{\xi}_{h}, \boldsymbol{\varphi}_{h}, \mu_{h}\right),\left(\boldsymbol{\xi}_{h},-\boldsymbol{\varphi}_{h},-\mu_{h}\right)\right) \geq \frac{12(1-\nu)}{E}\left\|\boldsymbol{\xi}_{h}\right\|_{0, \Omega}^{2}+\sum_{e \in \mathcal{E}} h_{e}\left\|\llbracket \boldsymbol{\varphi}_{h} \rrbracket\right\|_{0, e}^{2} .
$$


Furthermore, in order to control the $L^{2}(\Omega)$ norm of $\boldsymbol{\varphi}_{h} \in H_{h}^{\boldsymbol{\beta}}$, choosing its corresponding $\tilde{\boldsymbol{\tau}}_{h} \in \boldsymbol{H}_{h}^{\boldsymbol{\sigma}}$ from Lemma 3.1, using the Cauchy-Schwarz's and Young's inequalities and the continuity of $\mathcal{C}^{-1}$ (see $(3)$ ), gives

$$
\begin{aligned}
& a_{h}\left(\left(\boldsymbol{\xi}_{h}, \boldsymbol{\varphi}_{h}, \mu_{h}\right),\left(\tilde{\boldsymbol{\tau}}_{h}, \mathbf{0}, 0\right)\right)=\int_{\Omega} \mathcal{C}^{-1} \boldsymbol{\xi}_{h}: \tilde{\boldsymbol{\tau}}_{h}+\int_{\Omega} \boldsymbol{\varphi}_{h} \cdot \operatorname{div}\left(\tilde{\boldsymbol{\tau}}_{h}\right) \\
& \geq \frac{1}{2}\left\|\boldsymbol{\varphi}_{h}\right\|_{0, \Omega}^{2}-c_{i n f} \sum_{e \in \mathcal{E}} h_{e}\left\|\llbracket \boldsymbol{\varphi}_{h} \rrbracket\right\|_{0, e}^{2}-\frac{12(1+3 \nu)}{E}\left\|\boldsymbol{\xi}_{h}\right\|_{0, \Omega}\left\|\tilde{\boldsymbol{\tau}}_{h}\right\|_{0, \Omega} \\
& \geq \frac{1}{2}\left\|\boldsymbol{\varphi}_{h}\right\|_{0, \Omega}^{2}-c_{i n f} \sum_{e \in \mathcal{E}} h_{e}\left\|\llbracket \boldsymbol{\varphi}_{h} \rrbracket\right\|_{0, e}^{2}-\frac{6(1+3 \nu)}{E}\left\{\delta\left\|\boldsymbol{\xi}_{h}\right\|_{0, \Omega}^{2}+\frac{C_{\text {sup }}^{2}}{\delta}\left\|\boldsymbol{\varphi}_{h}\right\|_{0, \Omega}^{2}\right\},
\end{aligned}
$$

where $\delta>0$, and $C_{\text {sup }}, c_{\text {inf }}>0$ are the constants from Lemma 3.1. The choice $\delta=\frac{24(1+3 \nu) C_{s u p}^{2}}{E}$ yields

$$
a_{h}\left(\left(\boldsymbol{\xi}_{h}, \boldsymbol{\varphi}_{h}, \mu_{h}\right),\left(\tilde{\boldsymbol{\tau}}_{h}, \mathbf{0}, 0\right)\right) \geq \frac{1}{4}\left\|\boldsymbol{\varphi}_{h}\right\|_{0, \Omega}^{2}-c_{\text {inf }} \sum_{e \in \mathcal{E}} h_{e}\left\|\llbracket \boldsymbol{\varphi}_{h} \rrbracket\right\|_{0, e}^{2}-\frac{144(1+3 \nu)^{2} C_{s u p}^{2}}{E^{2}}\left\|\boldsymbol{\xi}_{h}\right\|_{0, \Omega}^{2} .
$$

Then, (45) and (47) suggest to define

$$
\left(\boldsymbol{\tau}_{h}, \boldsymbol{v}_{h}, q_{h}\right):=\left(\boldsymbol{\xi}_{h}+c_{0} \tilde{\boldsymbol{\tau}}_{h},-\boldsymbol{\varphi}_{h},-\mu_{h}\right),
$$

where $c_{0}>0$ is a positive constant to be chosen. This choice clearly satisfies

$$
\left\|\left(\boldsymbol{\tau}_{h}, \boldsymbol{v}_{h}, q_{h}\right)\right\|_{1} \leq\left(1+C_{\text {sup }} c_{0}\right)\left\|\left(\boldsymbol{\xi}_{h}, \boldsymbol{\varphi}_{h}, \mu_{h}\right)\right\|_{1} .
$$

In addition, using (45) and (47) one gets

$$
\begin{aligned}
& a_{h}\left(\left(\boldsymbol{\xi}_{h}, \boldsymbol{\varphi}_{h}, \mu_{h}\right),\left(\boldsymbol{\tau}_{h}, \boldsymbol{v}_{h}, q_{h}\right)\right) \geq\left(\frac{12(1-\nu)}{E}-\frac{c_{0} 144(1+3 \nu)^{2} C_{\text {sup }}^{2}}{E^{2}}\right)\left\|\boldsymbol{\xi}_{h}\right\|_{0, \Omega}^{2} \\
& +\frac{c_{0}}{4}\left\|\boldsymbol{\varphi}_{h}\right\|_{0, \Omega}^{2}+\left(1-c_{0} c_{i n f}\right) \sum_{e \in \mathcal{E}} h_{e}\left\|\llbracket \boldsymbol{\varphi}_{h} \rrbracket\right\|_{0, e}^{2}
\end{aligned}
$$

Hence, choosing

$$
c_{0}=\min \left\{\frac{6(1-\nu) E}{144(1+3 \nu)^{2} C_{\text {sup }}^{2}}, \frac{1}{4 c_{\text {inf }}}\right\}
$$

and using that $\varphi_{h}=\nabla \mu_{h}$, one obtains

$$
a_{h}\left(\left(\boldsymbol{\xi}_{h}, \boldsymbol{\varphi}_{h}, \mu_{h}\right),\left(\boldsymbol{\tau}_{h}, \boldsymbol{v}_{h}, q_{h}\right)\right) \geq C\left(\left\|\boldsymbol{\xi}_{h}\right\|_{0, \Omega}^{2}+\left\|\boldsymbol{\varphi}_{h}\right\|_{h, 0}^{2}\right) \geq \hat{C}\left\|\left(\boldsymbol{\xi}_{h}, \boldsymbol{\varphi}_{h}, \mu_{h}\right)\right\|_{1}^{2},
$$

where $\hat{C}>0$ is independent of $h$. Therefore, (44) is a consequence of (50) and (49), with $\tilde{\alpha}_{1}=\frac{\hat{C}}{1+C_{\text {sup }} c_{0}}$.

To study the well-posedness of (33), the space $\mathcal{X}_{h}$ is equipped with the following mesh-dependent norm

$$
\left\|\left(\boldsymbol{\tau}_{h}, \boldsymbol{v}_{h}, q_{h}, \boldsymbol{z}_{h}\right)\right\|_{h}^{2}:=\left\|\left(\boldsymbol{\tau}_{h}, \boldsymbol{v}_{h}, q_{h}\right)\right\|_{1}^{2}+\left\|\boldsymbol{z}_{h}\right\|_{0, \Omega}^{2},
$$

for all $\left(\boldsymbol{\tau}_{h}, \boldsymbol{v}_{h}, q_{h}, \boldsymbol{z}_{h}\right) \in \mathcal{X}_{h}$. The following result states a uniform inf-sup condition for $A_{h}$. 
Theorem 3.4 There exist $\tilde{\alpha}>0$, independent of $h$ and $t$, such that for all $\left(\boldsymbol{\xi}_{h}, \boldsymbol{\varphi}_{h}, \mu_{h}, \boldsymbol{\psi}_{h}\right) \in \mathcal{X}_{h}$ the following holds

$$
\sup _{\left(\boldsymbol{\tau}_{h}, \boldsymbol{v}_{h}, q_{h}, \boldsymbol{z}_{h}\right) \in \mathcal{X}_{h} \backslash\{\boldsymbol{0}\}} \frac{A_{h}\left(\left(\boldsymbol{\xi}_{h}, \boldsymbol{\varphi}_{h}, \mu_{h}, \boldsymbol{\psi}_{h}\right),\left(\boldsymbol{\tau}_{h}, \boldsymbol{v}_{h}, q_{h}, \boldsymbol{z}_{h}\right)\right)}{\left\|\left(\boldsymbol{\tau}_{h}, \boldsymbol{v}_{h}, q_{h}, \boldsymbol{z}_{h}\right)\right\|_{h}} \geq \tilde{\alpha}\left\|\left(\boldsymbol{\xi}_{h}, \boldsymbol{\varphi}_{h}, \mu_{h}, \boldsymbol{\psi}_{h}\right)\right\|_{h} .
$$

Consequently, there exists a unique $\left(\boldsymbol{\sigma}_{h}, \boldsymbol{\beta}_{h}, \omega_{h}, \boldsymbol{\gamma}_{h}\right) \in \mathcal{X}_{h}$ solution of the problem (33). Moreover, this solution satisfies

$$
\left\|\left(\boldsymbol{\sigma}_{h}, \boldsymbol{\beta}_{h}, \omega_{h}, \gamma_{h}\right)\right\|_{h} \leq \frac{1}{\tilde{\alpha}}\|g\|_{-1, \Omega} .
$$

Finally, if $(\boldsymbol{\sigma}, \boldsymbol{\beta}, \omega, \boldsymbol{\gamma})$, solution of $(11)$ is such that $\boldsymbol{\beta} \in H_{0}^{1}(\Omega)^{2}$, then the following Galerkin orthogonality holds

$$
A_{h}\left(\left(\boldsymbol{\sigma}-\boldsymbol{\sigma}_{h}, \boldsymbol{\beta}-\boldsymbol{\beta}_{h}, \omega-\omega_{h}, \boldsymbol{\gamma}-\boldsymbol{\gamma}_{h}\right),\left(\boldsymbol{\tau}_{h}, \boldsymbol{v}_{h}, q_{h}, \boldsymbol{z}_{h}\right)\right)=0 \quad \forall\left(\boldsymbol{\tau}_{h}, \boldsymbol{v}_{h}, q_{h}, \boldsymbol{z}_{h}\right) \in \mathcal{X}_{h} .
$$

Proof. The proof of (52) is reminiscent of the one from [6], Lemma B.1. Indeed, let $\left(\boldsymbol{\xi}_{h}, \boldsymbol{\varphi}_{h}, \mu_{h}, \boldsymbol{\psi}_{h}\right) \in$ $\mathcal{X}_{h}$. First, from Lemmas 3.2 and 3.3, using [16], or [13], Proposition 2.36, there exists $\left(\boldsymbol{\tau}_{h}, \boldsymbol{v}_{h}, q_{h}, \boldsymbol{z}_{h}\right) \in$ $\mathcal{X}_{h}$ such that

$$
\begin{gathered}
a_{h}\left(\left(\boldsymbol{\xi}_{h}, \boldsymbol{\varphi}_{h}, \mu_{h}\right),\left(\boldsymbol{\tau}_{h}, \boldsymbol{v}_{h}, q_{h}\right)\right)+b\left(\left(\boldsymbol{\xi}_{h}, \boldsymbol{\varphi}_{h}, \mu_{h}\right), \boldsymbol{z}_{h}\right)+b\left(\left(\boldsymbol{\tau}_{h}, \boldsymbol{v}_{h}, q_{h}\right), \boldsymbol{\psi}_{h}\right) \\
\geq \tilde{\gamma}\left\|\left(\boldsymbol{\xi}_{h}, \boldsymbol{\varphi}_{h}, \mu_{h}, \boldsymbol{\psi}_{h}\right)\right\|_{h}\left\|\left(\boldsymbol{\tau}_{h}, \boldsymbol{v}_{h}, q_{h}, \boldsymbol{z}_{h}\right)\right\|_{h},
\end{gathered}
$$

for some $\tilde{\gamma}>0$, independent of $h$ and $t$.

In addition, from the proof of Lemma 3.3 (cf. (48),(49) and (50)) there exists $\hat{\boldsymbol{\tau}}_{h} \in H_{h}^{\boldsymbol{\sigma}}$ such that

$$
a_{h}\left(\left(\boldsymbol{\xi}_{h}, \boldsymbol{\varphi}_{h}, \mu_{h}\right),\left(\hat{\boldsymbol{\tau}}_{h},-\boldsymbol{\varphi}_{h},-\mu_{h}\right)\right) \geq c\left\|\left(\boldsymbol{\xi}_{h}, \boldsymbol{\varphi}_{h}, \mu_{h}\right)\right\|_{1}\left\|\left(\hat{\boldsymbol{\tau}}_{h},-\boldsymbol{\varphi}_{h},-\mu_{h}\right)\right\|_{1} \geq 0 .
$$

Now, if one supposes that

$$
a_{h}\left(\left(\boldsymbol{\xi}_{h}, \boldsymbol{\varphi}_{h}, \mu_{h}\right),\left(\hat{\boldsymbol{\tau}}_{h},-\boldsymbol{\varphi}_{h},-\mu_{h}\right)\right)+\frac{t^{2}}{\kappa}\left\|\boldsymbol{\psi}_{h}\right\|_{0, \Omega}^{2} \geq \frac{\tilde{\gamma}^{2}}{4}\left\|\left(\boldsymbol{\xi}_{h}, \boldsymbol{\varphi}_{h}, \mu_{h}, \boldsymbol{\psi}_{h}\right)\right\|_{h}^{2},
$$

then,

$$
\begin{aligned}
A_{h}\left(\left(\boldsymbol{\xi}_{h}, \boldsymbol{\varphi}_{h}, \mu_{h}, \boldsymbol{\psi}_{h}\right),\left(\hat{\boldsymbol{\tau}}_{h},-\boldsymbol{\varphi}_{h},-\mu_{h}, \boldsymbol{\psi}_{h}\right)\right) & =a_{h}\left(\left(\boldsymbol{\xi}_{h}, \boldsymbol{\varphi}_{h}, \mu_{h}\right),\left(\hat{\boldsymbol{\tau}}_{h},-\boldsymbol{\varphi}_{h},-\mu_{h}\right)\right)+\frac{t^{2}}{\kappa}\left\|\boldsymbol{\psi}_{h}\right\|_{0, \Omega}^{2} \\
& \geq \frac{\tilde{\gamma}^{2}}{4}\left\|\left(\boldsymbol{\xi}_{h}, \boldsymbol{\varphi}_{h}, \mu_{h}, \boldsymbol{\psi}_{h}\right)\right\|_{h}^{2},
\end{aligned}
$$

and the result follows using that $\left\|\hat{\boldsymbol{\tau}}_{h}\right\|_{0, \Omega} \leq C_{0}\left\|\boldsymbol{\xi}_{h}\right\|_{0, \Omega}$ and $\tilde{\alpha}=\min \left\{1, C_{0}^{-1}\right\} \frac{\tilde{\gamma}^{2}}{4}$ (independent of $h$ and $t$ ). On the other hand, if one supposes that

$$
a_{h}\left(\left(\boldsymbol{\xi}_{h}, \boldsymbol{\varphi}_{h}, \mu_{h}\right),\left(\hat{\boldsymbol{\tau}}_{h},-\boldsymbol{\varphi}_{h},-\mu_{h}\right)\right)+\frac{t^{2}}{\kappa}\left\|\boldsymbol{\psi}_{h}\right\|_{0, \Omega}^{2} \leq \frac{\tilde{\gamma}^{2}}{4}\left\|\left(\boldsymbol{\xi}_{h}, \boldsymbol{\varphi}_{h}, \mu_{h}, \boldsymbol{\psi}_{h}\right)\right\|_{h}^{2},
$$


then the Cauchy-Schwarz inequality, (56), and $\frac{t^{2}}{\kappa} \leq 1$ give for any $\left(\boldsymbol{\tau}_{h}, \boldsymbol{v}_{h}, q_{h}, \boldsymbol{z}_{h}\right) \in \mathcal{X}_{h}$

$$
\begin{aligned}
\frac{t^{2}}{\kappa} \int_{\Omega} \boldsymbol{\psi}_{h} \cdot \boldsymbol{z}_{h} & \leq\left[\frac{t^{2}}{\kappa}\left\|\boldsymbol{\psi}_{h}\right\|_{0, \Omega}^{2} \frac{t^{2}}{\kappa}\left\|\boldsymbol{z}_{h}\right\|_{0, \Omega}^{2}\right]^{\frac{1}{2}} \leq\left[\frac{t^{2}}{\kappa}\left\|\boldsymbol{\psi}_{h}\right\|_{0, \Omega}^{2}\left\|\boldsymbol{z}_{h}\right\|_{0, \Omega}^{2}\right]^{\frac{1}{2}} \\
& \leq\left[\frac{\tilde{\gamma}^{2}}{4}\left\|\left(\boldsymbol{\xi}_{h}, \boldsymbol{\varphi}_{h}, \mu_{h}, \boldsymbol{\psi}_{h}\right)\right\|_{h}^{2}\left\|\left(\boldsymbol{\tau}_{h}, \boldsymbol{v}_{h}, q_{h}, \boldsymbol{z}_{h}\right)\right\|_{h}^{2}\right]^{\frac{1}{2}} .
\end{aligned}
$$

This implies that, for $\left(\boldsymbol{\tau}_{h}, \boldsymbol{v}_{h}, q_{h}, \boldsymbol{z}_{h}\right) \in \mathcal{X}_{h}$ satisfying (55), there holds

$$
\begin{aligned}
A_{h}\left(\left(\boldsymbol{\xi}_{h}, \boldsymbol{\varphi}_{h}, \mu_{h}, \boldsymbol{\psi}_{h}\right),\left(\boldsymbol{\tau}_{h}, \boldsymbol{v}_{h}, q_{h}, \boldsymbol{z}_{h}\right)\right) \geq & \tilde{\gamma}\left\|\left(\boldsymbol{\xi}_{h}, \boldsymbol{\varphi}_{h}, \mu_{h}, \boldsymbol{\psi}_{h}\right)\right\|_{h}\left\|\left(\boldsymbol{\tau}_{h}, \boldsymbol{v}_{h}, q_{h}, \boldsymbol{z}_{h}\right)\right\|_{h} \\
& -\left[\frac{\tilde{\gamma}^{2}}{4}\left\|\left(\boldsymbol{\xi}_{h}, \boldsymbol{\varphi}_{h}, \mu_{h}, \boldsymbol{\psi}_{h}\right)\right\|_{h}^{2}\left\|\left(\boldsymbol{\tau}_{h}, \boldsymbol{v}_{h}, q_{h}, \boldsymbol{z}_{h}\right)\right\|_{h}^{2}\right]^{\frac{1}{2}},
\end{aligned}
$$

which proves $(52)$ with $\tilde{\alpha}=\frac{\tilde{\gamma}}{2}$, again independent of $h$ and $t$. This proves the inf-sup condition (52) and the well-posedness of (33). The stability result (53) is a direct consequence of (52). Finally, (54) follows by noting that if $\boldsymbol{\beta} \in H_{0}^{1}(\Omega)^{2}$, then $\llbracket \boldsymbol{\beta} \rrbracket=\mathbf{0}$ a.e. over all the edges $e \in \mathcal{E}$.

\subsection{Error analysis}

We begin by introducing some notation. Let $(\boldsymbol{\sigma}, \boldsymbol{\beta}, \omega, \boldsymbol{\gamma}) \in \mathcal{X}$ and $\left(\boldsymbol{\sigma}_{h}, \boldsymbol{\beta}_{h}, \omega_{h}, \boldsymbol{\gamma}_{h}\right) \in \mathcal{X}_{h}$ be the unique solutions of (11) and (33), respectively. Hereafter, the individual error between $\boldsymbol{\sigma}$ and $\boldsymbol{\sigma}_{h}$ is denoted by $\boldsymbol{e}^{\boldsymbol{\sigma}}:=\boldsymbol{\sigma}-\boldsymbol{\sigma}_{h}$, and the errors $\boldsymbol{e}^{\boldsymbol{\beta}}, e^{\omega}$ and $\boldsymbol{e}^{\boldsymbol{\gamma}}$ are defined in an analogous way. The next result states a first error estimate for this method.

Theorem 3.5 Let us suppose that the solution of (11) satisfies $(\boldsymbol{\sigma}, \boldsymbol{\beta}, \omega, \boldsymbol{\gamma}) \in H^{2}(\Omega)^{2 \times 2} \times H_{0}^{1}(\Omega)^{2} \times$ $H^{2}(\Omega) \times H^{1}(\Omega)^{2}$. Then, there exists $C>0$, independent of $h$ and $t$, such that

$$
\left\|\left(\boldsymbol{e}^{\boldsymbol{\sigma}}, \boldsymbol{e}^{\boldsymbol{\beta}}, e^{\omega}\right)\right\|_{1}+t\left\|\boldsymbol{e}^{\boldsymbol{\gamma}}\right\|_{0, \Omega} \leq C h\left(\|\boldsymbol{\sigma}\|_{2, \Omega}+\|\boldsymbol{\beta}\|_{1, \Omega}+\|\omega\|_{2, \Omega}+t\|\boldsymbol{\gamma}\|_{1, \Omega}\right) .
$$

Proof. The error is split into discrete and interpolation errors as follows

$$
\begin{aligned}
\left(\boldsymbol{e}^{\boldsymbol{\sigma}}, \boldsymbol{e}^{\boldsymbol{\beta}}, e^{\omega}, \boldsymbol{e}^{\boldsymbol{\gamma}}\right)= & \left(\boldsymbol{\sigma}-I_{h}(\boldsymbol{\sigma}), \boldsymbol{\beta}-\Pi_{h, 0}(\boldsymbol{\beta}), \omega-I_{h}(\omega), \boldsymbol{\gamma}-\Pi_{h, 0}(\boldsymbol{\gamma})\right) \\
& +\left(I_{h}(\boldsymbol{\sigma})-\boldsymbol{\sigma}_{h}, \Pi_{h, 0}(\boldsymbol{\beta})-\boldsymbol{\beta}_{h}, I_{h}(\omega)-\omega_{h}, \Pi_{h, 0}(\boldsymbol{\gamma})-\gamma_{h}\right) \\
= & :\left(\boldsymbol{\eta}^{\boldsymbol{\sigma}}, \boldsymbol{\eta}^{\boldsymbol{\beta}}, \eta^{\omega}, \boldsymbol{\eta}^{\boldsymbol{\gamma}}\right)+\left(\boldsymbol{e}_{h}^{\boldsymbol{\sigma}}, \boldsymbol{e}_{h}^{\boldsymbol{\beta}}, e_{h}^{\omega}, \boldsymbol{e}_{h}^{\boldsymbol{\gamma}}\right) .
\end{aligned}
$$

First, (27)-(30), and the mesh regularity give

$$
\left\|\left(\boldsymbol{\eta}^{\boldsymbol{\sigma}}, \boldsymbol{\eta}^{\boldsymbol{\beta}}, \eta^{\omega}\right)\right\|_{1}+t\left\|\boldsymbol{\eta}^{\gamma}\right\|_{0, \Omega} \leq C h\left(\|\boldsymbol{\sigma}\|_{1, \Omega}+\|\boldsymbol{\beta}\|_{1, \Omega}+\|\omega\|_{2, \Omega}+t\|\boldsymbol{\gamma}\|_{1, \Omega}\right) .
$$

To bound the discrete error one starts noticing that Theorem 3.4 guarantees the existence of $\left(\boldsymbol{\tau}_{h}, \boldsymbol{v}_{h}, q_{h}, \boldsymbol{z}_{h}\right) \in$ $\mathcal{X}_{h}$ such that $\left\|\left(\boldsymbol{\tau}_{h}, \boldsymbol{v}_{h}, q_{h}, \boldsymbol{z}_{h}\right)\right\|_{h}=1$ and

$$
\tilde{\alpha}\left\|\left(\boldsymbol{e}_{h}^{\boldsymbol{\sigma}}, \boldsymbol{e}_{h}^{\boldsymbol{\beta}}, e_{h}^{\omega}, \boldsymbol{e}_{h}^{\boldsymbol{\gamma}}\right)\right\|_{h} \leq A_{h}\left(\left(\boldsymbol{e}_{h}^{\boldsymbol{\sigma}}, \boldsymbol{e}_{h}^{\boldsymbol{\beta}}, e_{h}^{\omega}, \boldsymbol{e}_{h}^{\boldsymbol{\gamma}}\right),\left(\boldsymbol{\tau}_{h}, \boldsymbol{v}_{h}, q_{h}, \boldsymbol{z}_{h}\right)\right) .
$$


Next, using the consistency of the method (cf. (54)) one obtains

$$
\begin{aligned}
\tilde{\alpha} & \left\|\left(\boldsymbol{e}_{h}^{\boldsymbol{\sigma}}, \boldsymbol{e}_{h}^{\boldsymbol{\beta}}, e_{h}^{\omega}, \boldsymbol{e}_{h}^{\boldsymbol{\gamma}}\right)\right\|_{h} \leq A_{h}\left(\left(\boldsymbol{e}_{h}^{\boldsymbol{\sigma}}, \boldsymbol{e}_{h}^{\boldsymbol{\beta}}, e_{h}^{\omega}, \boldsymbol{e}_{h}^{\boldsymbol{\gamma}}\right),\left(\boldsymbol{\tau}_{h}, \boldsymbol{v}_{h}, q_{h}, \boldsymbol{z}_{h}\right)\right) \\
= & A_{h}\left(\left(\boldsymbol{\eta}^{\boldsymbol{\sigma}}, \boldsymbol{\eta}^{\boldsymbol{\beta}}, \eta^{\omega}, \boldsymbol{\eta}^{\boldsymbol{\gamma}}\right),\left(\boldsymbol{\tau}_{h}, \boldsymbol{v}_{h}, q_{h}, \boldsymbol{z}_{h}\right)\right) \\
= & \int_{\Omega} \mathcal{C}^{-1} \boldsymbol{\eta}^{\boldsymbol{\sigma}}: \boldsymbol{\tau}_{h}+\int_{\Omega} \boldsymbol{\eta}^{\boldsymbol{\beta}} \cdot \operatorname{div}\left(\boldsymbol{\tau}_{h}\right)+\int_{\Omega} \boldsymbol{v}_{h} \cdot \operatorname{div}\left(\boldsymbol{\eta}^{\boldsymbol{\sigma}}\right)-\sum_{e \in \mathcal{E}} h_{e} \int_{e} \llbracket \boldsymbol{\eta}^{\boldsymbol{\beta}} \rrbracket \cdot \llbracket \boldsymbol{v}_{h} \rrbracket \\
& -\int_{\Omega} \boldsymbol{\eta}^{\boldsymbol{\gamma}} \cdot\left(\nabla q_{h}-\boldsymbol{v}_{h}\right)-\int_{\Omega}\left(\nabla \eta^{\omega}-\boldsymbol{\eta}^{\boldsymbol{\beta}}\right) \cdot \boldsymbol{z}_{h}+\frac{t^{2}}{\kappa} \int_{\Omega} \boldsymbol{\eta}^{\boldsymbol{\gamma}} \cdot \boldsymbol{z}_{h} .
\end{aligned}
$$

Now, since $\Pi_{h, 0}$ is the orthogonal projection with respect to the $L^{2}(\Omega)$ inner product it follows that

$$
\int_{\Omega} \boldsymbol{\eta}^{\boldsymbol{\beta}} \cdot \operatorname{div}\left(\boldsymbol{\tau}_{h}\right)=0, \int_{\Omega} \boldsymbol{\eta}^{\boldsymbol{\gamma}} \cdot\left(\nabla q_{h}-\boldsymbol{v}_{h}\right)=0, \int_{\Omega} \boldsymbol{\eta}^{\boldsymbol{\beta}} \cdot \boldsymbol{z}_{h}=0, \text { and } \frac{t^{2}}{\kappa} \int_{\Omega} \boldsymbol{\eta}^{\boldsymbol{\gamma}} \cdot \boldsymbol{z}_{h}=0 .
$$

The remaining terms are bounded one by one. The main tools are integration by parts, the mesh regularity, and the approximation properties (27)-(30):

$$
\begin{aligned}
\int_{\Omega} \mathcal{C}^{-1} \boldsymbol{\eta}^{\boldsymbol{\sigma}}: \boldsymbol{\tau}_{h} & \leq C\left\|\boldsymbol{\eta}^{\boldsymbol{\sigma}}\right\|_{0, \Omega}\left\|\boldsymbol{\tau}_{h}\right\|_{0, \Omega} \leq C h|\boldsymbol{\sigma}|_{1, \Omega}, \\
\int_{\Omega} \boldsymbol{v}_{h} \cdot \operatorname{div}\left(\boldsymbol{\eta}^{\boldsymbol{\sigma}}\right) & \leq C h\|\boldsymbol{\sigma}\|_{2, \Omega}, \\
\sum_{e \in \mathcal{E}} h_{e} \int_{e} \llbracket \boldsymbol{\eta}^{\boldsymbol{\beta}} \rrbracket \cdot \llbracket \boldsymbol{v}_{h} \rrbracket & \leq C\left\{\sum_{T \in \mathcal{T}_{h}} h_{T}\left\|\boldsymbol{\eta}^{\boldsymbol{\beta}}\right\|_{0, \partial T}^{2}\right\}^{\frac{1}{2}}\left\{\sum_{e \in \mathcal{E}} h_{e}\left\|\llbracket \boldsymbol{v}_{h} \rrbracket\right\|_{0, e}^{2}\right\}^{\frac{1}{2}} \leq C h\|\boldsymbol{\beta}\|_{1, \Omega}, \\
\int_{\Omega} \nabla \eta^{\omega} \cdot \boldsymbol{z}_{h} & \leq C h\|\omega\|_{2, \Omega} .
\end{aligned}
$$

The proof then follows from the triangle inequality and (58).

There are two terms missing from estimate (57), namely, the error in the divergence of $\boldsymbol{\sigma}$ and the error in the $L^{2}$-norm of $\gamma$ (i.e., without the parameter $t$ in front of it). The next result states an error estimate for a combination of these two terms.

Corollary 3.6 Under the hypothesis of Theorem 3.5, there exists a constant $C>0$, independent of $h$ and $t$, such that

$$
\left\|\operatorname{div}\left(\boldsymbol{e}^{\boldsymbol{\sigma}}\right)+\boldsymbol{e}^{\boldsymbol{\gamma}}\right\|_{0, \Omega} \leq C h\left(\|\boldsymbol{\sigma}\|_{2, \Omega}+\|\boldsymbol{\beta}\|_{1, \Omega}+\|\omega\|_{2, \Omega}+t\|\boldsymbol{\gamma}\|_{1, \Omega}\right) .
$$

Proof. Using the definition of method (33) it follows that

$$
\int_{\Omega}\left(\operatorname{div}\left(\boldsymbol{\sigma}_{h}\right)+\boldsymbol{\gamma}_{h}\right) \cdot \boldsymbol{v}_{h}=\sum_{e \in \mathcal{E}} h_{e} \int_{e} \llbracket \boldsymbol{\beta}_{h} \rrbracket \cdot \llbracket \boldsymbol{v}_{h} \rrbracket,
$$


for all $\boldsymbol{v}_{h} \in H_{h}^{\boldsymbol{\beta}}$. Then, taking $\boldsymbol{v}_{h}=\operatorname{div}\left(\boldsymbol{\sigma}_{h}\right)+\gamma_{h} \in H_{h}^{\boldsymbol{\beta}}$ and using the Cauchy-Schwarz inequality and (42) one gets

$$
\left\|\operatorname{div}\left(\boldsymbol{\sigma}_{h}\right)+\gamma_{h}\right\|_{0, \Omega}^{2} \leq C \sum_{e \in \mathcal{E}} h_{e}\left\|\llbracket \boldsymbol{\beta}_{h} \rrbracket\right\|_{0, e}^{2} .
$$

Then, using that $\operatorname{div}(\boldsymbol{\sigma})+\boldsymbol{\gamma}=\mathbf{0}$ in $\Omega, \llbracket \boldsymbol{\beta} \rrbracket=\mathbf{0}$ a.e. over all the edges $e \in \mathcal{E}$, the local trace result (21), and (57) it follows that

$$
\left\|\operatorname{div}\left(\boldsymbol{e}^{\boldsymbol{\sigma}}\right)+\boldsymbol{e}^{\boldsymbol{\gamma}}\right\|_{0, \Omega} \leq C h\left(\|\boldsymbol{\sigma}\|_{2, \Omega}+\|\boldsymbol{\beta}\|_{1, \Omega}+\|\omega\|_{2, \Omega}+t\|\gamma\|_{1, \Omega}\right)
$$

and the proof is finished.

Some comments on the last results are in order. The most important is that, even if the constants in the estimate are independent of $t$, the error estimate itself is not fully robust. This is due to the possible $t$-dependency of the norms on the right-hand-side of (57). More precisely, the norm $\|\boldsymbol{\sigma}\|_{2, \Omega}$ is, apriori, dependent on $t$ (the rest of the terms appearing in the right-hand-side of (57) and (65) can be bounded by $\|g\|_{0, \Omega}$ for a convex plate thanks to (14)). The main reason for this is the lack of an interpolation operator onto $H_{h}^{\boldsymbol{\beta}}$ that preseves the divergence. The existence of such an operator is a fundamental hypothesis in the analyses presented in [4],[3], and [11], and then the analyses of those references doesn't seem to be applicable to the present approach. The same comments are valid for the analysis presented in the next section. Nevertheless, it is worth mentioning that the numerical results indicate a robustness of the errors with respect to the value of $t$.

\section{A Method using linear continuous elements in all variables}

In this section we extend the ideas and results of the previous section to propose a method using the following choice of finite element spaces

$$
\begin{aligned}
H_{h}^{\boldsymbol{\sigma}} & :=\left\{\boldsymbol{\tau}_{h} \in X_{h, 1}^{2 \times 2}: \boldsymbol{\tau}_{h}=\boldsymbol{\tau}_{h}^{\mathrm{t}} \quad \text { in } \Omega\right\}, \\
H_{h}^{\boldsymbol{\beta}}=H_{h}^{\gamma} & :=X_{h, 1}^{2}, \\
H_{h}^{\omega} & :=X_{h, 1} \cap H_{0}^{1}(\Omega),
\end{aligned}
$$

and

$$
\mathcal{X}_{h, 1}:=H_{h}^{\sigma} \times H_{h}^{\boldsymbol{\beta}} \times H_{h}^{\omega} \times H_{h}^{\gamma} .
$$

Now, the analogous of Lemma 3.1 reads as follows: for every $\boldsymbol{v}_{h} \in X_{h, 1}^{2}$, there exists $\tilde{\boldsymbol{\tau}}_{h} \in H_{h}^{\sigma}$ such that

$$
\left\|\tilde{\boldsymbol{\tau}}_{h}\right\|_{1, \Omega} \leq C_{\text {sup }}\left\|\boldsymbol{v}_{h}\right\|_{0, \Omega}
$$

and

$$
\int_{\Omega} \operatorname{div}\left(\tilde{\boldsymbol{\tau}}_{h}\right) \cdot \boldsymbol{v}_{h} \geq \frac{1}{2}\left\|\boldsymbol{v}_{h}\right\|_{0, \Omega}^{2}-c_{i n f}\left\{\sum_{T \in \mathcal{T}_{h}} h_{T}^{2}\left\|\varepsilon\left(\boldsymbol{v}_{h}\right)\right\|_{0, T}^{2}+\sum_{e \in \mathcal{E}_{b}} h_{e}\left\|\boldsymbol{v}_{h}\right\|_{0, e}^{2}\right\},
$$

where the positive constants $C_{\text {sup }}$ and $c_{\text {inf }}$ are independent of $\boldsymbol{v}_{h}, h$, and, in particular, $t$. 
Then, with the aim of curing this inf-sup deficiency and obtaining estimates as those from Theorem 3.5, we propose the following method: Find $\left(\boldsymbol{\sigma}_{h}, \boldsymbol{\beta}_{h}, \omega_{h}, \gamma_{h}\right) \in \mathcal{X}_{h, 1}$ such that

$$
A_{h, 1}\left(\left(\boldsymbol{\sigma}_{h}, \boldsymbol{\beta}_{h}, \omega_{h}, \boldsymbol{\gamma}_{h}\right),\left(\boldsymbol{\tau}_{h}, \boldsymbol{v}_{h}, q_{h}, \boldsymbol{z}_{h}\right)\right)=-\int_{\Omega} g q_{h} \quad \forall\left(\boldsymbol{\tau}_{h}, \boldsymbol{v}_{h}, q_{h}, \boldsymbol{z}_{h}\right) \in \mathcal{X}_{h, 1},
$$

where the bilinear form $A_{h, 1}: \mathcal{X}_{h, 1} \times \mathcal{X}_{h, 1} \rightarrow \mathbb{R}$ is defined by

$$
\begin{gathered}
A_{h, 1}\left(\left(\boldsymbol{\sigma}_{h}, \boldsymbol{\beta}_{h}, \omega_{h}, \boldsymbol{\gamma}_{h}\right),\left(\boldsymbol{\tau}_{h}, \boldsymbol{v}_{h}, q_{h}, \boldsymbol{z}_{h}\right)\right):=A\left(\left(\boldsymbol{\sigma}_{h}, \boldsymbol{\beta}_{h}, \omega_{h}, \boldsymbol{\gamma}_{h}\right),\left(\boldsymbol{\tau}_{h}, \boldsymbol{v}_{h}, q_{h}, \boldsymbol{z}_{h}\right)\right) \\
-\sum_{T \in \mathcal{T}_{h}} h_{T}^{2} \int_{T} \boldsymbol{\varepsilon}\left(\boldsymbol{\beta}_{h}\right): \boldsymbol{\varepsilon}\left(\boldsymbol{v}_{h}\right)-\sum_{e \in \mathcal{E}_{b}} h_{e} \int_{e} \boldsymbol{\beta}_{h} \cdot \boldsymbol{v}_{h}-\sum_{e \in \mathcal{E}_{I}} h_{e} \int_{e} \llbracket \partial_{\boldsymbol{n}} \omega_{h} \rrbracket \llbracket \partial_{\boldsymbol{n}} q_{h} \rrbracket .
\end{gathered}
$$

Remark 4.1 Let, for every node $x_{i}$ of $\mathcal{T}_{h}, n_{i}$ be the number of triangles having $x_{i}$ as a node. Then, for a piecewise smooth function $v$, one defines its Oswald quasi-interpolate as follows (cf. [20])

$$
\pi_{h}^{\star} v\left(x_{i}\right)=\left.\frac{1}{n_{i}} \sum_{T: x_{i} \in T} v\right|_{T}\left(x_{i}\right) .
$$

Using this mapping, one can follow the same steps as in [10], Theorem 2.2, to prove that there exists a constant $c>0$, independent of $h$, such that

$$
\sum_{e \in \mathcal{E}_{I}} h_{e}\left\|\llbracket \partial_{\boldsymbol{n}} \mu_{h} \rrbracket\right\|_{0, e}^{2} \geq c\left\|\nabla \mu_{h}-\pi_{h}^{\star}\left(\nabla \mu_{h}\right)\right\|_{0, \Omega}^{2} \geq c\left\|\nabla \mu_{h}-\Pi_{h, 1}\left(\nabla \mu_{h}\right)\right\|_{0, \Omega}^{2} .
$$

As it will become clear in the proof of stability below, this fact explains the need to add the term containing the jumps of $\omega_{h}$ to the formulation.

To perform the analysis, let $\mathcal{Y}_{h}:=H_{h}^{\boldsymbol{\sigma}} \times H_{h}^{\boldsymbol{\beta}} \times H_{h}^{\omega}$; the bilinear form $b$ is once again given by (37) while the bilinear form $a_{h}: \mathcal{Y}_{h} \times \mathcal{Y}_{h} \rightarrow \mathbb{R}$ is given by

$$
\begin{gathered}
a_{h}\left(\left(\boldsymbol{\sigma}_{h}, \boldsymbol{\beta}_{h}, \omega_{h}\right),\left(\boldsymbol{\tau}_{h}, \boldsymbol{v}_{h}, q_{h}\right)\right)=\int_{\Omega} \mathcal{C}^{-1} \boldsymbol{\sigma}_{h}: \boldsymbol{\tau}_{h}+\int_{\Omega} \boldsymbol{\beta}_{h} \cdot \operatorname{div}\left(\boldsymbol{\tau}_{h}\right)+\int_{\Omega} \operatorname{div}\left(\boldsymbol{\sigma}_{h}\right) \cdot \boldsymbol{v}_{h} \\
-\sum_{T \in \mathcal{T}_{h}} h_{T}^{2} \int_{T} \boldsymbol{\varepsilon}\left(\boldsymbol{\beta}_{h}\right): \boldsymbol{\varepsilon}\left(\boldsymbol{v}_{h}\right)-\sum_{e \in \mathcal{E}_{b}} h_{e} \int_{e} \boldsymbol{\beta}_{h} \cdot \boldsymbol{v}_{h}-\sum_{e \in \mathcal{E}_{I}} h_{e} \int_{e} \llbracket \partial_{\boldsymbol{n}} \omega_{h} \rrbracket \llbracket \partial_{\boldsymbol{n}} q_{h} \rrbracket .
\end{gathered}
$$

The following norms will be the ones used in the analysis:

$$
\begin{aligned}
\left\|\boldsymbol{v}_{h}\right\|_{h, 1}^{2} & :=\left\|\boldsymbol{v}_{h}\right\|_{0, \Omega}^{2}+\sum_{T \in \mathcal{T}_{h}} h_{T}^{2}\left\|\boldsymbol{\varepsilon}\left(\boldsymbol{v}_{h}\right)\right\|_{0, T}^{2}+\sum_{e \in \mathcal{E}_{b}} h_{e}\left\|\boldsymbol{v}_{h}\right\|_{0, e}^{2}, \\
\left\|\left(\boldsymbol{\tau}_{h}, \boldsymbol{v}_{h}, q_{h}\right)\right\|_{2}^{2} & :=\left\|\boldsymbol{\tau}_{h}\right\|_{0, \Omega}^{2}+\left\|\boldsymbol{v}_{h}\right\|_{h, 1}^{2}+\left\|\nabla q_{h}\right\|_{0, \Omega}^{2} \\
\left\|\left(\boldsymbol{\tau}_{h}, \boldsymbol{v}_{h}, q_{h}, \boldsymbol{z}_{h}\right)\right\|_{\star}^{2} & :=\left\|\left(\boldsymbol{\tau}_{h}, \boldsymbol{v}_{h}, q_{h}\right)\right\|_{2}^{2}+\left\|\boldsymbol{z}_{h}\right\|_{0, \Omega}^{2} .
\end{aligned}
$$

Then, the analogous of Lemmas 3.2 and 3.3 from the previous section read as follows. 
Lemma 4.2 There exists $\tilde{\alpha}_{0}>0$, independent of $h$ such that

$$
\sup _{\left(\boldsymbol{\tau}_{h}, \boldsymbol{v}_{h}, q_{h}\right) \in \mathcal{Y}_{h} \backslash\{0\}} \frac{b\left(\left(\boldsymbol{\tau}_{h}, \boldsymbol{v}_{h}, q_{h}\right), \boldsymbol{z}_{h}\right)}{\left\|\left(\boldsymbol{\tau}_{h}, \boldsymbol{v}_{h}, q_{h}\right)\right\|_{2}} \geq \tilde{\alpha}_{0}\left\|\boldsymbol{z}_{h}\right\|_{0, \Omega},
$$

for all $\boldsymbol{z}_{h} \in H^{\gamma}$. Moreover, the discrete kernel of the bilinear form $b$ is given by

$$
\tilde{\boldsymbol{V}}_{h}=\left\{\left(\boldsymbol{\tau}_{h}, \boldsymbol{v}_{h}, q_{h}\right) \in \mathcal{Y}_{h}: \boldsymbol{v}_{h}=\Pi_{h, 1}\left(\nabla q_{h}\right) \text { in } \Omega\right\} .
$$

In addition, there exists $\tilde{\alpha}_{1}>0$, independent of $h$, such that for all $\left(\boldsymbol{\xi}_{h}, \boldsymbol{\varphi}_{h}, \mu_{h}\right) \in \tilde{\boldsymbol{V}}_{h}$ the following holds

$$
\sup _{\left(\boldsymbol{\tau}_{h}, \boldsymbol{v}_{h}, q_{h}\right) \in \tilde{\boldsymbol{V}}_{h} \backslash\{0\}} \frac{a_{h}\left(\left(\boldsymbol{\xi}_{h}, \boldsymbol{\varphi}_{h}, \mu_{h}\right),\left(\boldsymbol{\tau}_{h}, \boldsymbol{v}_{h}, q_{h}\right)\right)}{\left\|\left(\boldsymbol{\tau}_{h}, \boldsymbol{v}_{h}, q_{h}\right)\right\|_{2}} \geq \tilde{\alpha}_{1}\left\|\left(\boldsymbol{\xi}_{h}, \boldsymbol{\varphi}_{h}, \mu_{h}\right)\right\|_{2} .
$$

Proof. The proof of the inf-sup condition (79) is similar to the proof of (40). The only difference now being that the analogous to (42) uses a local inverse inequality. The characterisation of $\tilde{\boldsymbol{V}}_{h}$ is an immediate consequence of the definition of $\Pi_{h, 1}$. Finally, to prove $(80)$, let $\left(\boldsymbol{\xi}_{h}, \boldsymbol{\varphi}_{h}, \mu_{h}\right) \in \tilde{\boldsymbol{V}}_{h}$. Then, as in the proof of Lemma 3.3 , there exists $\hat{\tau}_{h} \in H_{h}^{\sigma}$ such that

$$
\begin{aligned}
a_{h}\left(\left(\boldsymbol{\xi}_{h}, \boldsymbol{\varphi}_{h}, \mu_{h}\right),\left(\hat{\boldsymbol{\tau}}_{h},-\boldsymbol{\varphi}_{h},-\mu_{h}\right)\right) \geq & c\left(\left\|\boldsymbol{\xi}_{h}\right\|_{0, \Omega}^{2}+\left\|\boldsymbol{\varphi}_{h}\right\|_{h, 1}^{2}+\sum_{e \in \mathcal{E}_{I}} h_{e}\left\|\llbracket \partial_{\boldsymbol{n}} \mu_{h} \rrbracket\right\|_{0, e}^{2}\right)^{\frac{1}{2}} \\
& \left(\left\|\hat{\boldsymbol{\tau}}_{h}\right\|_{0, \Omega}^{2}+\left\|\boldsymbol{\varphi}_{h}\right\|_{h, 1}^{2}+\sum_{e \in \mathcal{E}_{I}} h_{e}\left\|\llbracket \partial_{\boldsymbol{n}} \mu_{h} \rrbracket\right\|_{0, e}^{2}\right)^{\frac{1}{2}},
\end{aligned}
$$

where $c>0$ does not depend on $\left(\boldsymbol{\xi}_{h}, \boldsymbol{\varphi}_{h}, \mu_{h}\right)$ or $h$. Finally, since $\boldsymbol{\varphi}_{h}=\Pi_{h, 1}\left(\nabla \mu_{h}\right)$, then using (76) one obtains

$$
\begin{gathered}
\left\|\boldsymbol{\varphi}_{h}\right\|_{h, 1}^{2}+\sum_{e \in \mathcal{E}_{I}} h_{e}\left\|\llbracket \partial_{\boldsymbol{n}} \mu_{h} \rrbracket\right\|_{0, e}^{2} \geq \frac{1}{2}\left\|\boldsymbol{\varphi}_{h}\right\|_{h, 1}^{2}+\frac{1}{2}\left\|\Pi_{h, 1}\left(\nabla \mu_{h}\right)\right\|_{0, \Omega}^{2}+c\left\|\nabla \mu_{h}-\Pi_{h, 1}\left(\nabla \mu_{h}\right)\right\|_{0, \Omega}^{2} \\
\geq \frac{1}{2}\left\|\boldsymbol{\varphi}_{h}\right\|_{h, 1}^{2}+\min \left\{\frac{1}{2}, c\right\}\left\|\nabla \mu_{h}\right\|_{0, \Omega}^{2}
\end{gathered}
$$

and the proof follows.

As a consequence of these inf-sup conditions, and following the same steps as in the proof of Theorem 3.4, one can prove the following result stating the stability of the method with constants independent of the plate thickness $t$.

Theorem 4.3 There exist $\tilde{\alpha}>0$, independent of $h$ and $t$, such that for all $\left(\boldsymbol{\xi}_{h}, \boldsymbol{\varphi}_{h}, \mu_{h}, \boldsymbol{\psi}_{h}\right) \in \mathcal{X}_{h, 1}$,

$$
\sup _{\left(\boldsymbol{\tau}_{h}, \boldsymbol{v}_{h}, q_{h}, \boldsymbol{z}_{h}\right) \in \mathcal{X}_{h, 1} \backslash\{\mathbf{0}\}} \frac{A_{h, 1}\left(\left(\boldsymbol{\xi}_{h}, \boldsymbol{\varphi}_{h}, \mu_{h}, \boldsymbol{\psi}_{h}\right),\left(\boldsymbol{\tau}_{h}, \boldsymbol{v}_{h}, q_{h}, \boldsymbol{z}_{h}\right)\right)}{\left\|\left(\boldsymbol{\tau}_{h}, \boldsymbol{v}_{h}, q_{h}, \boldsymbol{z}_{h}\right)\right\|_{\star}} \geq \tilde{\alpha}\left\|\left(\boldsymbol{\xi}_{h}, \boldsymbol{\varphi}_{h}, \mu_{h}, \boldsymbol{\psi}_{h}\right)\right\|_{\star} .
$$


Consequently, there exists a unique $\left(\boldsymbol{\sigma}_{h}, \boldsymbol{\beta}_{h}, \omega_{h}, \gamma_{h}\right) \in \mathcal{X}_{h, 1}$, solution of the problem (75).

In addition, if $(\boldsymbol{\sigma}, \boldsymbol{\beta}, \omega, \boldsymbol{\gamma})$ is the solution of $(11)$ is such that $\boldsymbol{\beta} \in H_{0}^{1}(\Omega)^{2}$ and $\omega \in H^{2}(\Omega)$, then the following holds

$$
A_{h, 1}\left(\left(\boldsymbol{\sigma}-\boldsymbol{\sigma}_{h}, \boldsymbol{\beta}-\boldsymbol{\beta}_{h}, \omega-\omega_{h}, \boldsymbol{\gamma}-\boldsymbol{\gamma}_{h}\right),\left(\boldsymbol{\tau}_{h}, \boldsymbol{v}_{h}, q_{h}, \boldsymbol{z}_{h}\right)\right)=-\sum_{T \in \mathcal{T}_{h}} h_{T}^{2} \int_{T} \varepsilon(\boldsymbol{\beta}): \varepsilon\left(\boldsymbol{v}_{h}\right),
$$

for all $\left(\boldsymbol{\tau}_{h}, \boldsymbol{v}_{h}, q_{h}, \boldsymbol{z}_{h}\right) \in \mathcal{X}_{h, 1}$.

Finally, we state the main convergence result of this section.

Theorem 4.4 Let us suppose that the solution of (11) satisfies $(\boldsymbol{\sigma}, \boldsymbol{\beta}, \omega, \boldsymbol{\gamma}) \in H^{2}(\Omega)^{2 \times 2} \times\left(H^{2}(\Omega) \cap\right.$ $\left.H_{0}^{1}(\Omega)\right)^{2} \times H^{2}(\Omega) \times H^{1}(\Omega)^{2}$. Then, there exists $C>0$, independent of $h$ and $t$, such that

$$
\begin{aligned}
\left\|\left(\boldsymbol{e}^{\boldsymbol{\sigma}}, \boldsymbol{e}^{\boldsymbol{\beta}}, e^{\omega}, \boldsymbol{e}^{\boldsymbol{\gamma}}\right)\right\|_{\star} \leq C h\left(\|\boldsymbol{\sigma}\|_{2, \Omega}+\|\boldsymbol{\beta}\|_{2, \Omega}+\|\omega\|_{2, \Omega}+\|\boldsymbol{\gamma}\|_{1, \Omega}\right), \\
\left\|\operatorname{div}(\boldsymbol{\sigma})-\Pi_{h, 1}\left(\operatorname{div}\left(\boldsymbol{\sigma}_{h}\right)\right)\right\|_{0, \Omega} \leq C h\left(\|\boldsymbol{\sigma}\|_{2, \Omega}+\|\boldsymbol{\beta}\|_{2, \Omega}+\|\omega\|_{2, \Omega}+\|\boldsymbol{\gamma}\|_{1, \Omega}\right) .
\end{aligned}
$$

Proof. As before, the error is split into interpolation and discrete errors. For this case, all variables are interpolated using their respective Scott-Zhang interpolates. Using (27) and (28) the interpolation error satisfies

$$
\left\|\left(\boldsymbol{\eta}^{\boldsymbol{\sigma}}, \boldsymbol{\eta}^{\boldsymbol{\beta}}, \eta^{\omega}, \boldsymbol{\eta}^{\gamma}\right)\right\|_{\star} \leq C h\left(\|\boldsymbol{\sigma}\|_{1, \Omega}+\|\boldsymbol{\beta}\|_{1, \Omega}+\|\omega\|_{2, \Omega}+\|\boldsymbol{\gamma}\|_{1, \Omega}\right) .
$$

To bound the discrete error, from Theorem 4.3 there exists $\left(\boldsymbol{\tau}_{h}, \boldsymbol{v}_{h}, q_{h}, \boldsymbol{z}_{h}\right) \in \mathcal{X}_{h, 1}$ satisfying $\left\|\left(\boldsymbol{\tau}_{h}, \boldsymbol{v}_{h}, q_{h}, \boldsymbol{z}_{h}\right)\right\|_{\star}=$ 1 and $\tilde{\alpha}\left\|\left(\boldsymbol{e}_{h}^{\boldsymbol{\sigma}}, \boldsymbol{e}_{h}^{\boldsymbol{\beta}}, e_{h}^{\omega}, \boldsymbol{e}_{h}^{\boldsymbol{\gamma}}\right)\right\|_{\star} \leq A_{h, 1}\left(\left(\boldsymbol{e}_{h}^{\boldsymbol{\sigma}}, \boldsymbol{e}_{h}^{\boldsymbol{\beta}}, e_{h}^{\omega}, \boldsymbol{e}_{h}^{\boldsymbol{\gamma}}\right),\left(\boldsymbol{\tau}_{h}, \boldsymbol{v}_{h}, q_{h}, \boldsymbol{z}_{h}\right)\right)$. Then, using (82) and the definition of the bilinear form $A_{h, 1}$, one gets

$$
\begin{aligned}
\tilde{\alpha} & \left\|\left(\boldsymbol{e}_{h}^{\boldsymbol{\sigma}}, \boldsymbol{e}_{h}^{\boldsymbol{\beta}}, e_{h}^{\omega}, \boldsymbol{e}_{h}^{\boldsymbol{\gamma}}\right)\right\|_{\star} \leq A_{h, 1}\left(\left(\boldsymbol{\eta}^{\boldsymbol{\sigma}}, \boldsymbol{\eta}^{\boldsymbol{\beta}}, \eta^{\omega}, \boldsymbol{\eta}^{\boldsymbol{\gamma}}\right),\left(\boldsymbol{\tau}_{h}, \boldsymbol{v}_{h}, q_{h}, \boldsymbol{z}_{h}\right)\right)-\sum_{T \in \mathcal{T}_{h}} h_{T}^{2} \int_{T} \boldsymbol{\varepsilon}(\boldsymbol{\beta}): \boldsymbol{\varepsilon}\left(\boldsymbol{v}_{h}\right) \\
= & \int_{\Omega} \mathcal{C}^{-1} \boldsymbol{\eta}^{\boldsymbol{\sigma}}: \boldsymbol{\tau}_{h}+\int_{\Omega} \boldsymbol{\eta}^{\boldsymbol{\beta}} \cdot \operatorname{div}\left(\boldsymbol{\tau}_{h}\right)+\int_{\Omega} \boldsymbol{v}_{h} \cdot \operatorname{div}\left(\boldsymbol{\eta}^{\boldsymbol{\sigma}}\right)-\int_{\Omega} \boldsymbol{\eta}^{\boldsymbol{\gamma}} \cdot\left(\nabla q_{h}-\boldsymbol{v}_{h}\right) \\
& -\int_{\Omega}\left(\nabla \eta^{\omega}-\boldsymbol{\eta}^{\boldsymbol{\beta}}\right) \cdot \boldsymbol{z}_{h}+\frac{t^{2}}{\kappa} \int_{\Omega} \boldsymbol{\eta}^{\boldsymbol{\gamma}} \cdot \boldsymbol{z}_{h}-\sum_{T \in \mathcal{T}_{h}} h_{T}^{2} \int_{T} \boldsymbol{\varepsilon}\left(\boldsymbol{\eta}^{\boldsymbol{\beta}}\right): \boldsymbol{\varepsilon}\left(\boldsymbol{v}_{h}\right) \\
& -\sum_{e \in \mathcal{E}_{I}} h_{e} \int_{e} \llbracket \partial_{\boldsymbol{n}} \eta^{\omega} \rrbracket \llbracket \partial_{\boldsymbol{n}} q_{h} \rrbracket-\sum_{T \in \mathcal{T}_{h}} h_{T}^{2} \int_{T} \boldsymbol{\varepsilon}(\boldsymbol{\beta}): \boldsymbol{\varepsilon}\left(\boldsymbol{v}_{h}\right) .
\end{aligned}
$$

Again, each term is bounded separately. The main ingredients are the Cauchy-Schwarz's inequality, an inverse inequality, bounds (27)-(28), and the local trace result (21):

$$
\begin{array}{ll}
\int_{\Omega} \mathcal{C}^{-1} \boldsymbol{\eta}^{\boldsymbol{\sigma}}: \boldsymbol{\tau}_{h} \leq C h\|\boldsymbol{\sigma}\|_{1, \Omega}, & \int_{\Omega} \boldsymbol{\eta}^{\boldsymbol{\beta}} \cdot \operatorname{div}\left(\boldsymbol{\tau}_{h}\right) \leq C h\|\boldsymbol{\beta}\|_{2, \Omega}, \\
\int_{\Omega} \boldsymbol{v}_{h} \cdot \operatorname{div}\left(\boldsymbol{\eta}^{\boldsymbol{\sigma}}\right) \leq C h\|\boldsymbol{\sigma}\|_{2, \Omega}, & \int_{\Omega} \boldsymbol{\eta}^{\boldsymbol{\gamma}} \cdot\left(\nabla q_{h}-\boldsymbol{v}_{h}\right) \leq C h\left\|_{\boldsymbol{\gamma}}\right\|_{1, \Omega}, \\
\int_{\Omega}\left(\nabla \eta^{\omega}-\boldsymbol{\eta}^{\boldsymbol{\beta}}\right) \cdot \boldsymbol{z}_{h} \leq C h\left(\|\omega\|_{2, \Omega}+\|\boldsymbol{\beta}\|_{1, \Omega}\right), & \frac{t^{2}}{\kappa} \int_{\Omega} \boldsymbol{\eta}^{\boldsymbol{\gamma}} \cdot \boldsymbol{z}_{h} \leq C h t^{2}\|\boldsymbol{\gamma}\|_{1, \Omega}, \\
\sum_{T \in \mathcal{T}_{h}} h_{T}^{2} \int_{T} \boldsymbol{\varepsilon}\left(\boldsymbol{\eta}^{\boldsymbol{\beta}}\right): \boldsymbol{\varepsilon}\left(\boldsymbol{v}_{h}\right) \leq C h\|\boldsymbol{\beta}\|_{1, \Omega}, & \sum_{T \in \mathcal{T}_{h}} h_{T}^{2} \int_{T} \boldsymbol{\varepsilon}(\boldsymbol{\beta}): \boldsymbol{\varepsilon}\left(\boldsymbol{z}_{h}\right) \leq C h\|\boldsymbol{\beta}\|_{1, \Omega}, \\
\sum_{e \in \mathcal{E}_{I}} h_{e} \int_{e} \llbracket \partial_{\boldsymbol{n}} \eta^{\omega} \rrbracket \llbracket \partial_{\boldsymbol{n}} q_{h} \rrbracket \leq C h\|\omega\|_{2, \Omega}, &
\end{array}
$$


and (83) follows using the triangle inequality. Estimate (84) is proved as in Corollary 3.6. In fact, for every $\boldsymbol{v}_{h} \in H_{h}^{\boldsymbol{\beta}}(74)$ yields

$$
\int_{\Omega}\left(\operatorname{div}\left(\boldsymbol{\sigma}_{h}\right)+\boldsymbol{\gamma}_{h}\right) \cdot \boldsymbol{v}_{h}=\sum_{T \in \mathcal{T}_{h}} h_{T}^{2} \int_{T} \varepsilon\left(\boldsymbol{\beta}_{h}\right): \varepsilon\left(\boldsymbol{v}_{h}\right)+\sum_{e \in \mathcal{E}_{b}} h_{e} \int_{e} \boldsymbol{\beta}_{h} \cdot \boldsymbol{v}_{h}
$$

and then it is enough to consider $\boldsymbol{v}_{h}=\Pi_{h, 1}\left(\operatorname{div}\left(\boldsymbol{\sigma}_{h}\right)\right)+\gamma_{h} \in H_{h}^{\boldsymbol{\beta}}$ and proceed as in the proof of Corollary 3.6.

\section{$5 \quad$ Numerical experiments}

In this section we report some numerical experiments which confirm the theoretical results provided in this work. The methods presented in the previous sections have been implemented using a code written in FEniCS. Some comments on the computational cost are in order. In the case of method (33), using a combination of piecewise linears and piecewise constants, method (33) leads to a linear system of, asymptotically, 12 times the number of vertices of the triangulation $\mathcal{T}_{h}$. This is computationally competitive with the previously existing alternatives [3] and [4]. This economy of unknowns is even more noticeable when the method (74) is used. In this latter case, the number of unknowns is asymptotically equal to 8 times the number of vertices.

\subsection{A problem with an analytical solution}

We first report the numerical results on the same experiment from [4]. Similar numerical results have been obtained for other test cases as well, but we prefer to restrict our presentation to this representative benchmark. We have considered an isotropic and homogeneous plate $\Omega=(0,1)^{2}$ clamped on the whole boundary for which the analytical solution is explicitly known. We choose the load $g$ as

$$
\begin{array}{r}
g(x, y)=\frac{E}{12\left(1-\nu^{2}\right)}\left[12 y(y-1)\left(5 x^{2}-5 x+1\right)\left(2 y^{2}(y-1)^{2}+x(x-1)\left(5 y^{2}-5 y+1\right)\right)\right. \\
\left.+12 x(x-1)\left(5 y^{2}-5 y+1\right)\left(2 x^{2}(x-1)^{2}+y(y-1)\left(5 x^{2}-5 x+1\right)\right)\right] .
\end{array}
$$

With this load, the exact solution of the plate problem is given by

$$
\begin{aligned}
\omega(x, y) & =\frac{1}{3} x^{3}(x-1)^{3} y^{3}(1-y)^{3} \\
& -\frac{2 t^{2}}{5(1-\nu)}\left[y^{3}(1-y)^{3} x(x-1)\left(5 x^{2}-5 x+1\right)+x^{3}(x-1)^{3} y(y-1)\left(5 y^{2}-5 y+1\right)\right], \\
\boldsymbol{\beta} & =\left(\begin{array}{c}
y^{3}(y-1)^{3} x^{2}(x-1)^{2}(2 x-1) \\
x^{3}(x-1)^{3} y^{2}(y-1)^{2}(2 y-1)
\end{array}\right) .
\end{aligned}
$$

The material constants have been chosen: $E=1, \nu=0.3$ and the shear correction factor has been taken as $k=5 / 6$. 
We start presenting the results obtained with method (33), using a combination of piecewise linear and piecewise constant interpolations. The method was implemented and the errors were computed using the sequence of meshes from Figure 1. We have computed the following quantities:

$$
e_{1}:=\left\|\boldsymbol{e}^{\boldsymbol{\sigma}}\right\|_{\operatorname{div}, \Omega}, e_{2}:=\left\|\boldsymbol{e}^{\boldsymbol{\beta}}\right\|_{h, 0}, e_{3}:=\left|e^{\omega}\right|_{1, \Omega}, e_{4}:=\left\|\boldsymbol{e}^{\boldsymbol{\gamma}}\right\|_{0, \Omega}
$$

These quantities are larger than the norm in which the error estimates (57) and (65) are stated, but we have chosen to plot them since they, in particular, imply the validation of the convergence estimates in the last sections. Calculations using $t=0.01$ were performed, and the results are depicted in Figure 2, where we observe that all quantities converge to zero as predicted by the theory. Moreover, in Figure 3 we plot the different contour plots of different variables in the finest mesh of the sequence. Finally, our aim is to study the robustness of the error in the $\|\cdot\|_{h}$-norm, defined by (51). For this, we computed this norm of the error for the same sequence of meshes and values of $t$ ranging from $t=0.1$ to $t=10^{-6}$. The results are depicted in Figure 4 where a very robust behaviour of the error can be observed, showing almost $t$-independent values.

We then repeat the same experiment using method (74), using piecewise linear interpolations for all variables. For the same sequence of meshes from Figure 1 we computed the $\|\cdot\|_{\star}$-norm of the error, where the norm is defined in (78). In Figure 5 we depict the behaviour of this error as $h$ tends to 0 and for different values of $t$. We observe that this norm tends to zero as predicted by the theory, and moreover it presents a very robust behaviour with respect to the value of $t$, showing results which, as in the errors for method (33), are virtually $t$-independent.
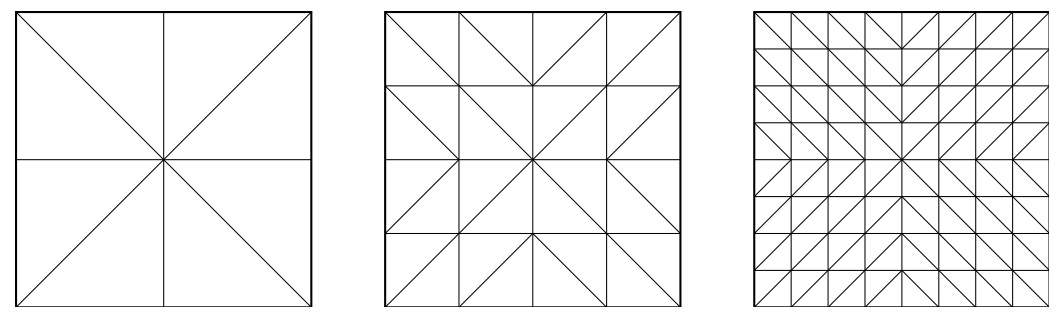

Figure 1: Sequence of uniform meshes on square plate.

Finally, to assess the presence, or lack of, locking in our method, we have repeated the experience carried out in the recent work [15], Section 5.3. For this, we have fixed a mesh from the sequence displayed in Figure 1, containing approximately 500 elements, solved the problem on it for different values of $t$, and have measured the maximum discrete displacement and compared it to the maximum exact displacement (measured in all of the nodes of the mesh). The results are provided in Table 1, where we can observe that both methods show a robust behaviour with respect to $t$.

\subsection{Assessing the lack of locking: a uniformily loaded plate}

In this section we repeat the numerical experiment from [11], Section 6.1. For this, we consider the square plate $\Omega=(-5,5)^{2}, E=10920, \nu=0.3, k=5 / 6$, impose homogeneous Dirichlet boundary 


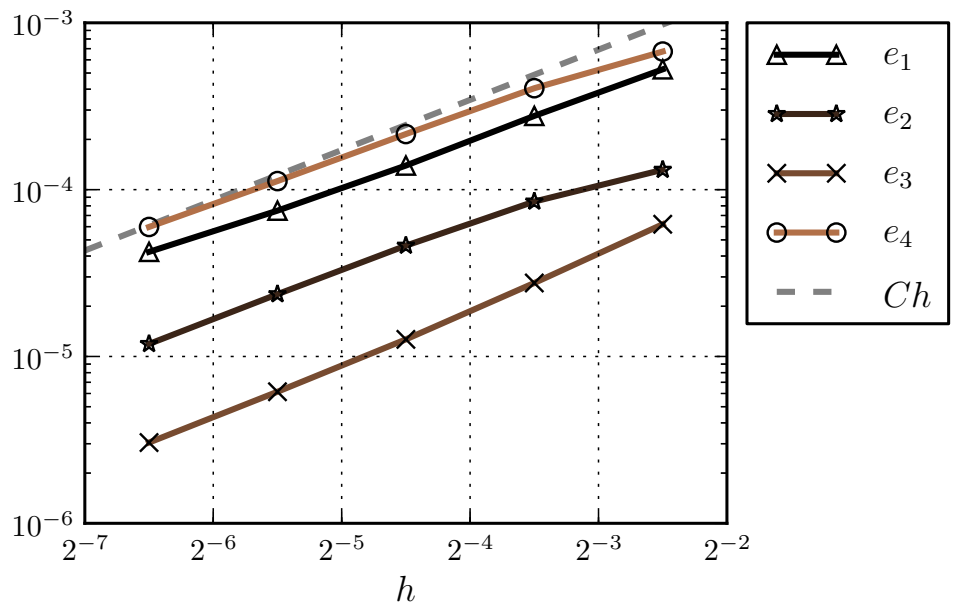

Figure 2: Convergence of the components of the energy-norm for thickness $t=10^{-2}$, for the $\mathbb{P}_{1} / \mathbb{P}_{0}$ method (33).

Table 1: Example 1: $\max \left|\omega_{h}\right| / \max |\omega|$ for different thicknesses $t$, on a fixed mesh.

\begin{tabular}{ccccccc}
\hline Method $\backslash t$ & 0.1 & $10^{-2}$ & $10^{-3}$ & $10^{-4}$ & $10^{-5}$ & $10^{-6}$ \\
\hline $\mathbb{P}_{1} / \mathbb{P}_{0}$ method (33) & 0.9405 & 0.9209 & 0.9206 & 0.9206 & 0.9206 & 0.9206 \\
$\mathbb{P}_{1} / \mathbb{P}_{1}$ method (74) & 0.8910 & 0.8872 & 0.8871 & 0.8871 & 0.8871 & 0.8871 \\
\hline
\end{tabular}

conditions on the whole boundary for both $\boldsymbol{\beta}$ and $\omega$, and impose the uniform loading $g=1$ in $\Omega$. We have solved this problem using the present approach and the non-conforming method from [2], which is known to be a locking-free finite element method. We have fixed a mesh of the same type as those from Figure 1, containing 12 divisions in each direction, and have solved the problem using the present methods (33) and (74), and the method from [2], on the same mesh. In Table 2 we report the scaled displacement in the centre of the plate, i.e., $10 \cdot \frac{E}{12 *\left(1-\nu^{2}\right)} \cdot \omega_{h}(0,0)$, for all methods. For comparison we have also included the results provided in [11]. The results show a very good agreement, and as well a good agreement with the exact scaled value reported in [11] which is $10 \cdot \frac{E}{12 *\left(1-\nu^{2}\right)} \cdot \omega(0,0)=126.5$. In particular, they show a robust bahavior with respect to the plate thickness $t$. Also, for this problem the tensor $\boldsymbol{\sigma}$ is diagonal (due to the symmetry of the problem), and then we have reported the scaled values for $-100 \cdot \boldsymbol{\sigma}_{11 h}(0,0)$ for the same different thickness, and compare them to the values reported in [11]. The results are reported in Table 3. Again, a good agreement can be observed, and also a good agreement with the exact scaled value reported in [11] which is $-100 \cdot \boldsymbol{\sigma}_{11}(0,0)=231$. 

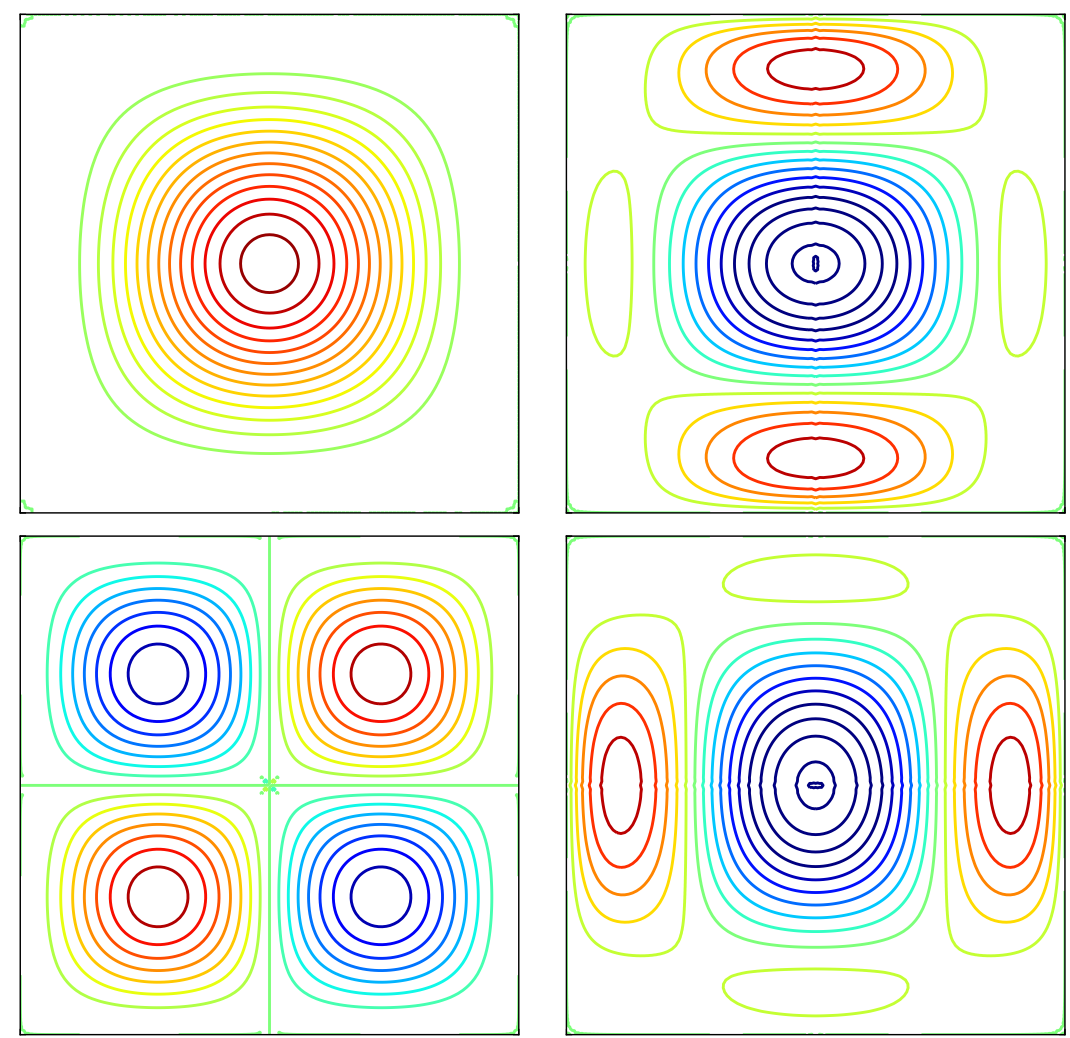

Figure 3: Approximations on finest mesh $(128 \times 128)$ for $t=0.01$, of transversal displacement $\omega_{h}$ (top left), bending moment $\sigma_{11 h}$ (top right), bending moment $\sigma_{12 h}$ (bottom left) and $\sigma_{22 h}$ (bottom right).

\section{References}

[1] M. Amara, D. Capatina-Papaghiuc and A. Chatti: New locking-free mixed method for the Reissner- Mindlin thin plate model. SIAM Journal of Numerical Analysis, vol. 40, 1561-1582, 2002.

[2] D.N. Arnold And R.S. Falk: A uniformly accurate finite element method for the ReissnerMindlin plate. SIAM Journal of Numerical Analysis, vol. 26, 6, 1276-1290, 1989.

[3] E. Behrens and J. Guzman: A new family of mixed methods for the Reissner-Mindling plate model based on a system of first-order equations. Journal of Scientific Computing, vol. 49, 137-166, 2011.

[4] L. Beirão DA Veiga, D. Mora And R. Rodríguez: Numerical analysis of a locking-free mixed finite element method for a bending moment formulation of Reissner-Mindlin plate model. Numerical Methods for Partial Differential Equations, vol. 29, 40-63, 2013. 


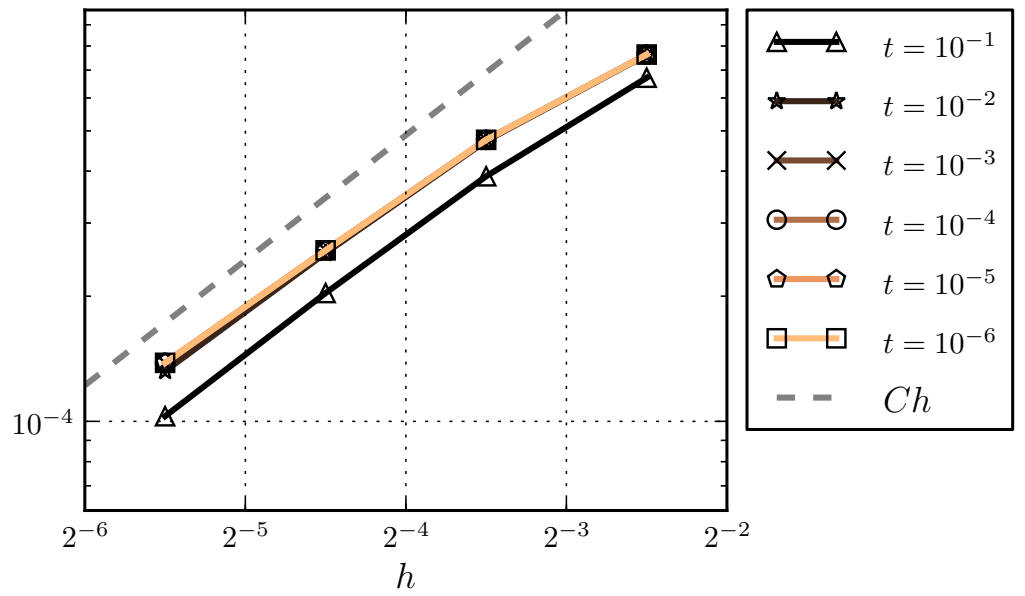

Figure 4: Independence of energy-error of thickness $t$ for the $\mathbb{P}_{1} / \mathbb{P}_{0} \operatorname{method}(33)$.

Table 2: Example 2: $10 \cdot \frac{E}{12 *\left(1-\nu^{2}\right)} \cdot \omega_{h}(0,0)$ for different methods and thicknesses $t$.

\begin{tabular}{ccccccc}
\hline Method $\backslash t$ & 0.1 & $10^{-2}$ & $10^{-3}$ & $10^{-4}$ & $10^{-5}$ & $10^{-6}$ \\
\hline $\mathbb{P}_{1} / \mathbb{P}_{0}$ method (33) & 126.88 & 126.60 & 126.59 & 126.59 & 126.59 & 126.59 \\
$\mathbb{P}_{1} / \mathbb{P}_{1}$ method (74) & 131.03 & 130.77 & 130.76 & 130.76 & 130.76 & 130.76 \\
Arnold-Falk [2] & 126.22 & 125.93 & 125.92 & 125.92 & 125.92 & 125.92 \\
Reference [11] & 130.6 & & & 130.4 & & 130.4 \\
\hline
\end{tabular}

[5] P. Bochev, C. Dohrmann and M. Gunzburger: Stabilisation of low-order mixed finite elements for the Stokes equations. SIAM J. Numer. Anal. vol. 44(1) 82-101, 2006.

[6] D. Braess And C. BlÖmer: A multigrid method for a parameter dependent problem in solid mechanics. Numerische Mathematik, vol. 57, 747-761, 1990.

[7] F. Brezzi And M. Fortin: Mixed and Hybrid Finite Element Methods. Springer-Verlag, 1991.

[8] F. Brezzi, M. Fortin, And R. Stenberg: Error analysis of mixed-interpolated elements for Reissner-Mindlin plates. Math. Models Methods Appl. Sci. Vol. 1 (2), 125-151, 1991.

[9] F. Brezzi And M. Fortin: A minimal stabilisation procedure for mixed finite element methods. Numer. Math., vol. 89 (2001) ,457-492, 2001.

[10] E. Burman: A unified analysis for conforming and nonconforming stabilised finite element methods using interior penalty. SIAM J. Numer. Anal., vol. 43(5), 2012-2033, 2005. 


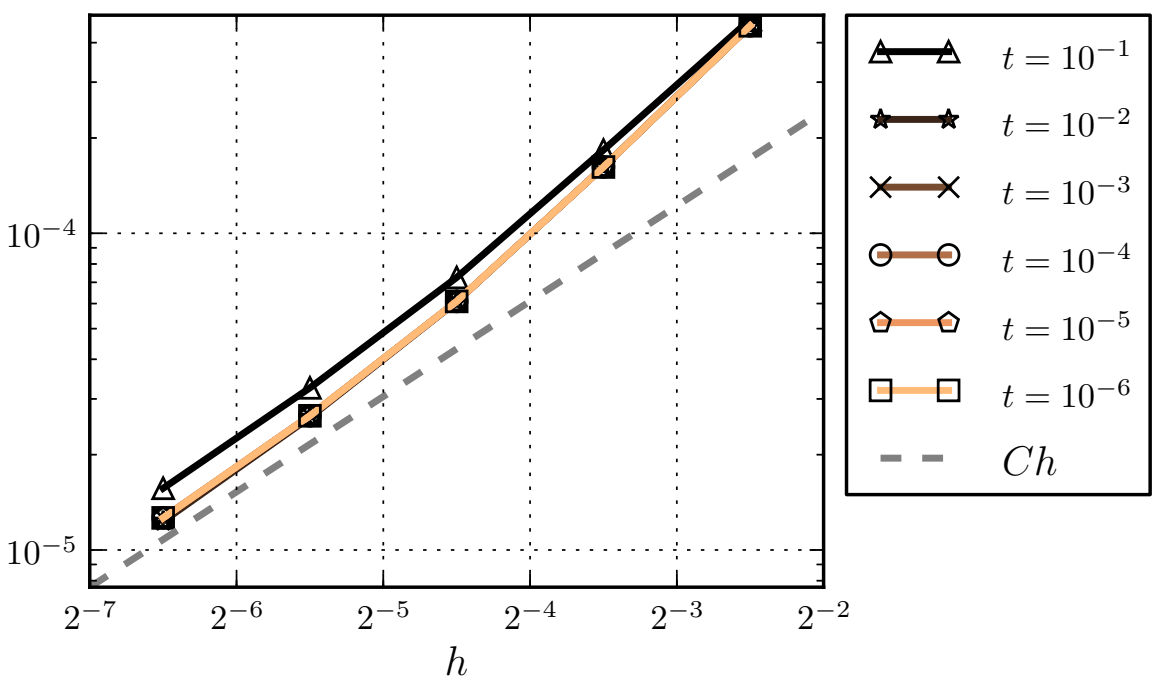

Figure 5: Independence of energy-error of thickness $t$ for the $\mathbb{P}_{1} / \mathbb{P}_{1} \operatorname{method}(74)$.

Table 3: Example 2: $100 \cdot \boldsymbol{\sigma}_{11 h}(0,0)$ for different methods and thicknesses $t$.

\begin{tabular}{ccccccc}
\hline Method $\backslash t$ & 0.1 & $10^{-2}$ & $10^{-3}$ & $10^{-4}$ & $10^{-5}$ & $10^{-6}$ \\
\hline $\mathbb{P}_{1} / \mathbb{P}_{0}$ method $(33)$ & 205.21 & 205.04 & 205.04 & 205.04 & 2.0504 & 205.04 \\
$\mathbb{P}_{1} / \mathbb{P}_{1}$ method (74) & 234.21 & 234.13 & 234.13 & 234.13 & 234.13 & 234.13 \\
Reference [1] & 240.5 & & & 240.5 & & 240.5 \\
\hline
\end{tabular}

[11] C. Carstensen, X. Xie, G. Yu And T. Zhou: A priori and a posterior analysis for a lockingfree low order quadrilateral hybrid finite element for Reissner-Mindlin plates. Comput. Methods Appl. Mech. Engrg., vol. 200, 1161-1175, 2011.

[12] R. DurÁn And E. Liberman: On mixed finite elements methods for the Reissner-Mindlin plate model. Mathematics of Computation, vol. 58, 561-573, 1992.

[13] A. Ern and J.-L. Guermond: Theory and Practice of Finite Element. Springer-Verlag, 2004.

[14] R. FALK: Finite Elements for the Reissner-Mindlin problem in Mixed Finite Elements, Compatibility Conditions, and Applications. Lecture Notes in Mathematics, Springer-Verlag, vol. 1939, 195-232, 2008.

[15] P. Hansbo And M. LARson: Locking free quadrilateral continuous/discontinuous finite element methods for the Reissner-Mindlin plate. Computer Methods in Applied Mechanics and Engineering, vol. 269, 381-393, 2014. 
[16] V. Girault and P.A. Raviart: Finite Element Methods for Navier-Stokes Equations: Theory and Algorithms. Springer Series in Computational Mathematics, 1986.

[17] T.J.R. Hughes And L.P. Franca: A mixed finite element formulation for Reissner-Mindlin plate theory: Uniform convergence of all Higher-Order spaces. Comput. Methods Appl. Mech. Engrg., vol. 67, 223-240, 1988.

[18] R. Kounia: On stabilised finite element methods for the Reissner-Mindlin plate model. International Journal for Numerical Methods in Engineering, vol. 74, 8, pp. 1314-1328, 2008.

[19] C. Lovadina: Analysis of a mixed finite element method for the Reissner-Mindlin plate problems. Computer Methods in Applied Mechanics and Engineering, vol. 163, pp. 71-85, 1998.

[20] P. Oswald: On a BPX-preconditioner for P1 elements, Tech. Report, FSU Jena, 1991.

[21] J. SchÖBERl AND R. Stenberg: Multigrid methods for a stabilised Reissner-Mindlin plate formulation. SIAM J. Numer. Anal., vol. 47(4), 2735-2751, 2009.

[22] R. Stenberg: A new finite element formulation for the plate bending problem. In Asymptotic methods for elastic structures (Lisbon, 1993), pp. 209-221, de Gruyter, Berlin, 1995. 